\title{
The Plethysm Technique Applied to the Classification of Nuclear States
}

\author{
J.A. Castilho Alcarás \\ Instituto de Física Teórica, UNESP, 01405-900, São Paulo, Brazil \\ J. Tambergs, T. Krasta, J.Ruža, \\ Nuclear Research Center, LV-2169, Salaspils, Latvia \\ and O. Katkevičius \\ Institute of Theoretical Physics and Astronomy, 2600 Vilnius, Lithuania
}

Received on 7 August, 2001

\begin{abstract}
A short summary of the theory of symmetric group and symmetric functions needed to follow the theory of Schur functions and plethysms is presented. One then defines plethysm, gives its properties and presents a procedure for its calculation. Finally, some aplications in atomic physics and nuclear structure are given.
\end{abstract}

\section{Introduction}

The plethysm operation was introduced by Littlewood[14] in 1936 as a third type of Schur function $[5,6]$ multiplication and only later on it has received this name.

Plethysms play a fundamental role in Physics whenever one applies group theory and can lead to remarkable simplification in the theory of complex spectra.

Although plethysms have been extensively studied by matematicians in the past [12]-[20] and even nowadays, surprisingly, with rare exceptions $[2,3,35,38]$ this powerful technique has been unrecognized by physicists.

The main reason of this work is to present this powerful technique to the theoretical physicists comunity and show how it can be very useful. Basically its utility relies in the determination of the branching rules of irreducible representations (irreps) of a continuous group when considered as irreps of one of its subgroups, a very common situation when one applies group theory, in particular in the classification of states of a physical system of identical particles.

\section{Schur Functions and Plethysms}

In this section we will initially introduce the basic notions of the symmetric group $S(n)$ needed to the understanding of the notation used in the plethysm theory. Later we will define plethysm, expose its properties, give general results and show how is it possible to compute plethysms of a given degree exploiting these general results and properties.

\section{II.1 Basic Notions of Symmetric Group and Schur Functions}

The symmetric group $S(n)$ is the group of permutations of $n$ objects and has $n$ ! elements. Each element is denoted by

$$
P=\left(\begin{array}{lllll}
1 & 2 & 3 & \cdots & \mathrm{n} \\
\mathrm{p}_{1} & \mathrm{p}_{2} & \mathrm{p}_{3} & \cdots & \mathrm{p}_{n}
\end{array}\right)
$$

with the meaning that the element 1 is replaced by $p_{1}$, the element 2 by $p_{2}$ and so on, $p_{1}, p_{2}, \ldots, p_{n}$ being a permutation of $1,2, \ldots, n$.

A permutation that changes $m \leq n$ object ciclicaly is called cicle of length $m$. Permutations with the same cicle structure belong to the same conjugation class. By this reason the classes of $S(n)$ are labeled by the cicle structure of their elements. In this way, a generic class $(\rho)$ is written as $(\rho) \equiv\left(1^{\rho_{1}}, 2^{\rho_{2}}, \ldots\right)$ with the meaning that their elements have $\rho_{1}$ cicles of length $1, \rho_{2}$ cicles of length 2 , and so on.

A partition $[\lambda]$ of $n$ is a set of $n$ integers $[\lambda] \equiv$ $\left[\lambda_{1}, \lambda_{2}, \ldots, \lambda_{n}\right]$ satisfying the relations

$$
\lambda_{1} \geq \lambda_{2} \geq \ldots \geq \lambda_{n}
$$

with

$$
\lambda_{1}+\lambda_{2}+\ldots+\lambda_{n}=n .
$$

In general one omits the $\lambda_{i}$ 's equal to zero and exponents the repeated ones. As an example [31] $\equiv[310 \ldots . .0]$, 
$\left[2^{2} 1^{3}\right] \equiv[221110 \ldots 0]$. We will denote by Greek letter a generic partition and by latin letter a particular one. As example, $[n] \equiv[n 0 \ldots 0],[p, q] \equiv[p, q, 0 \ldots 0]$.

To a given partition $[\lambda]$ one associates a Young diagram that consists in a figure with $\lambda_{1}$ boxes in the first line, $\lambda_{2}$ in the second, and so on. Due to this association, its is usual to refer to the $\lambda_{i}$ 's as the rows of $[\lambda]$.

When one fills these diagrams with the numbers $1,2, \ldots, n$ in such a way that the numbers in the boxes grow from left to right and from up to down they are called standard (or regular) Young tableau. When in the filling process the numbers in the rows only not decrease being allowed to repeat but in the columns they continue to grow, the tableau is called strict-column tableau.

Given the partition $[\lambda]=\left[\lambda_{1}, \lambda_{2}, \ldots, \lambda_{p}, 0, \ldots, 0\right]$ one defines its conjugate partition by

$$
[\widetilde{\lambda}]=\left[p^{\lambda_{p}},(p-1)^{\lambda_{p-1}-\lambda_{p}}, \ldots, 2^{\lambda_{2}-\lambda_{3}}, 1^{\lambda_{1}-\lambda_{2}}\right] .
$$

The Young diagram of $[\widetilde{\lambda}]$ is obtained from the one of $[\lambda]$ by interchanging rows by columns.
Given $n$ quantities $\alpha_{1}, \alpha_{2}, \ldots \alpha_{n}$ it is possible to define three types of symmetric functions built with them[1]: $a_{r}, h_{r}$ and $s_{r}$.

The functions $a_{i}$ are those that appear in the expansion of the polynomial with roots $\alpha_{1}, \alpha_{2}, \ldots \alpha_{n}$ as

$$
\prod_{i=1}^{n}\left(x-\alpha_{i}\right)=\sum_{r=0}^{n}(-)^{r} a_{r} x^{n-r}
$$

The first three of them are given by

$$
a_{1}=\sum_{i} \alpha_{i} ; \quad a_{2}=\sum_{i<j} \alpha_{i} \alpha_{j} ; \quad a_{3}=\sum_{i<j<k} \alpha_{i} \alpha_{j} \alpha_{k},
$$

that is, each $a_{i}$ is the sum of all linearly independent products of $r$ quantities $\alpha$ without repeated indices.

The functions $h_{r}$ are defined by the expansion

$$
\frac{1}{\prod_{i=1}^{n}\left(1-\alpha_{i} x\right)}=\sum_{r=0}^{\infty} h_{r} x^{r}
$$

resulting that each $h_{r}$ is the sum of all linearly independent products of $r$ quantities $\alpha$ allowing for repeatings. It follows from (4)- (6) that the first $h_{r}$ 's are

$$
\begin{aligned}
& h_{0}=1 ; \quad h_{1}=a_{1}=\sum_{i} \alpha_{i} ; \quad h_{2}=a_{1}^{2}-a_{2}=\sum_{i} \alpha_{i}^{2}+\sum_{i<j} \alpha_{i} \alpha_{j}, \\
& h_{3}=a_{3}-2 a_{1} a_{2}+a_{1}^{3}=\sum_{i<j<k} \alpha_{i} \alpha_{j} \alpha_{k}+\sum_{i<j}\left(\alpha_{i} \alpha_{j}^{2}+\alpha_{i}^{2} \alpha_{j}\right)+\sum_{i} \alpha_{i}^{3} .
\end{aligned}
$$

The functions $s_{r}$ are defined as the sums of the $r$-products of the $\alpha$ 's 1.e.,

$$
s_{r}=\sum_{i=1}^{n}\left(\alpha_{i}\right)^{r}
$$

Given a matrix $A$ of dimension $n \times n$ one defines its immanant $|A|^{[\lambda]}$ by the relation

$$
|A|^{[\lambda]}=\sum_{P} \chi^{[\lambda]}(P) a_{1 p_{1}} a_{2 p_{2}} \ldots a_{n p_{n}}
$$

where $\chi^{[\lambda]}(P)$ is the character of the permutation $P$ (with components $p_{1}, p_{2}, \ldots, p_{n}$ ) in the irrep $[\lambda]$ of $S(n)$.

Since in the irrep $\left[1^{n}\right]$ the character of a given permutation is its parity, $|A|^{\left[1^{n}\right]}$ is the determinant of $A$. Similarly, $|A|^{[n]}$ is its permanent.

The Schur function $\{\lambda\}$ of degree $r=\lambda_{1}+\lambda_{2}+\lambda_{3}+\ldots+\lambda_{n}$ in the quantities $\alpha_{1}, \ldots, \alpha_{n}$ is defined in terms of immanants as

$$
\{\lambda\}=\frac{1}{r !}\left|Z_{r}\right|^{[\lambda]}
$$

where $Z$ is the matrix 


$$
Z_{r}=\left(\begin{array}{llllllll}
\mathrm{s}_{1} & 1 & 0 & 0 & \ldots & \ldots & \ldots & 0 \\
\mathrm{~s}_{2} & \mathrm{~s}_{1} & 2 & 0 & \ldots & \ldots & \ldots & 0 \\
\mathrm{~s}_{3} & \mathrm{~s}_{2} & \mathrm{~s}_{1} & 3 & 0 & \ldots & \ldots & 0 \\
\ldots & \ldots & \ldots & . . & \ldots & \ldots & \ldots & 0 \\
\mathrm{~s}_{r-1} & \mathrm{~s}_{r-2} & \ldots & \ldots & \ldots & \ldots & \mathrm{s}_{1} & \mathrm{r}-1 \\
\mathrm{~s}_{r} & \mathrm{~s}_{r-1} & \ldots & \ldots & \ldots & \ldots & \mathrm{s}_{2} & \mathrm{~s}_{1}
\end{array}\right) .
$$

For the symmetric and antisymmetric partitions the Schur functions assume very simple expressions, namely,

$$
\{r\}=h_{r} \text { and }\left\{1^{r}\right\}=a_{r}
$$

As example, we give below the explicit expressions of the Schur functions of degree $\mathrm{r}=3$ :

$$
\begin{aligned}
\{3\} & =\frac{1}{6}\left(s_{1}^{3}+3 s_{1} s_{2}+2 s_{3}\right)=\sum_{i<j<k} \alpha_{i} \alpha_{j} \alpha_{k}+\sum_{i<j}\left(\alpha_{i} \alpha_{j}^{2}+\alpha_{i}^{2} \alpha_{j}\right)+\sum_{i} \alpha_{i}^{3} \equiv h_{3}, \\
\{21\} & =\frac{1}{3}\left(s_{1}^{3}-s_{3}\right)=2 \sum_{i<j<k} \alpha_{i} \alpha_{j} \alpha_{k}+\sum_{i<j}\left(\alpha_{i} \alpha_{j}^{2}+\alpha_{i}^{2} \alpha_{j}\right), \\
\left\{1^{3}\right\} & =\frac{1}{6}\left(s_{1}^{3}-3 s_{1} s_{2}+2 s_{3}\right)=\sum_{i<j<k} \alpha_{i} \alpha_{j} \alpha_{k} \equiv a_{3} .
\end{aligned}
$$

Using the explicit expressions of immanants in the definition of the Schur function one obtains the relation

$$
\{\lambda\}=\frac{1}{r !} \sum_{\rho} n(\rho) \chi^{[\lambda]}(\rho) s_{1}^{\rho_{1}} s_{2}^{\rho_{2}} \ldots
$$

which gives the fundamental link between Schur functions and characters of the symmetric group. In the equation above the sum is over the classes $(\rho) \equiv$ $\left(1^{\rho_{1}}, 2^{\rho_{2}}, \ldots\right)$ and $n(\rho)$ is the number elements of class $(\rho)$.

Schur functions of degree $r^{\prime}$ can be "multiplied" by Schur functions of degree $r^{\prime \prime}$ in 3 different ways and in each of them the "product" is expressed as a linear combination of Schur functions all of the same degree $r^{\prime \prime \prime}$.

These 3 types of multiplication are:

1) Inner(or direct) product: $\left\{\lambda^{\prime}\right\} \times\left\{\lambda^{\prime \prime}\right\}=$ $\sum_{\lambda^{\prime \prime \prime}} \alpha\left(\left\{\lambda^{\prime}\right\} \times\left\{\lambda^{\prime \prime}\right\} \rightarrow\left\{\lambda^{\prime \prime \prime}\right\}\right)\left\{\lambda^{\prime \prime \prime}\right\}$,

2) Outer product: $\left\{\lambda^{\prime}\right\}\left\{\lambda^{\prime \prime}\right\}=\sum_{\lambda^{\prime \prime \prime}} \alpha\left(\left\{\lambda^{\prime}\right\}\left\{\lambda^{\prime \prime}\right\} \rightarrow\right.$ $\left.\left\{\lambda^{\prime \prime \prime}\right\}\right)\left\{\lambda^{\prime \prime \prime}\right\}$,

3) Plethysm: $\{\lambda\} \otimes\left\{\lambda^{\prime \prime}\right\}=\sum_{\lambda^{\prime \prime \prime}} \alpha\left(\{\lambda\} \otimes\left\{\lambda^{\prime \prime}\right\} \rightarrow\right.$ $\left.\left\{\lambda^{\prime \prime \prime}\right\}\right)\left\{\lambda^{\prime \prime \prime}\right\}$, where $\alpha(\ldots)$ is a non-negative integer denoting the multiplicity of $\left\{\lambda^{\prime \prime \prime}\right\}$ in the expansion. For clarity we attach to it an argument denoting the kind of operation that produced it.

In the inner product the degrees of the Schur functions involved are all equal, i.e., $r^{\prime \prime \prime}=r^{\prime}=r^{\prime \prime}=n$, and the expansion coefficients $\alpha$ are the coefficients of reduction of the Kronecker product of $S(n)$ irreps $\left[\lambda^{\prime}\right]$ and $\left[\lambda^{\prime \prime}\right]$

In the outer product one has $r^{\prime \prime \prime}=r^{\prime}+r^{\prime \prime}$ and the coefficients $\alpha$ are obtained simply by making the product of a Schur function in variables $\left(x_{1}, x_{2}, \ldots, x_{n^{\prime}}\right)$ by another in variables $\left(y_{1}, y_{2}, \ldots, y_{n^{\prime \prime}}\right)$ and expressing it as a linear combination of Schur functions in variables $\left(z_{1}, z_{2}, \ldots, z_{n^{\prime \prime \prime}}\right)$ with $z_{i}=x_{i}$ for $1 \leq i \leq n^{\prime}$ and $z_{n^{\prime}+i}=y_{i}$ for $1 \leq i \leq n^{\prime \prime}$. Littlewood obtained a procedure to find the coefficients of the outer product known in the literature as "Littlewood's rules".

One finds in the literature[1],[3],[37] tables of outer products for $n^{\prime}+n^{\prime \prime}=1,2, \ldots, 14$. Tradicionally such tables are organized as the table given below for $n^{\prime}+n^{\prime \prime}=$ 5 .

\begin{tabular}{|l|l|l|l|l|l|l|l|l|}
\cline { 2 - 8 } \multicolumn{1}{c|}{} & $\{5\}$ & $\{41\}$ & $\{32\}$ & $\left\{31^{2}\right\}$ & $\left\{2^{2} 1\right\}$ & $\left\{21^{3}\right\}$ & $\left\{1^{5}\right\}$ & \\
\hline$\{4\}\{1\}$ & 1 & 1 & & & & & & $\left\{1^{4}\right\}\{1\}$ \\
\hline$\{31\}\{1\}$ & & 1 & 1 & 1 & & & & $\left\{21^{2}\right\}\{1\}$ \\
\hline$\left\{2^{2}\right\}\{1\}$ & & & 1 & & 1 & & & $\left\{2^{2}\right\}\{1\}$ \\
\hline$\{3\}\{2\}$ & 1 & 1 & 1 & & & & & $\left\{1^{3}\right\}\left\{1^{2}\right\}$ \\
\hline$\{21\}\{2\}$ & & 1 & 1 & 1 & 1 & & & $\{21\}\left\{1^{2}\right\}$ \\
\hline$\left\{1^{3}\right\}\{2\}$ & & & & 1 & & 1 & & $\{3\}\left\{1^{2}\right\}$ \\
\hline
\end{tabular}

Table I: All outer products that produce Schur functions of degree 5 . 
The numbers in the internal part of the table give the multiplicity of the Schur functions $\left\{\lambda^{\prime \prime \prime}\right\}$ that appear in the decomposition of the outer product $\left\{\lambda^{\prime}\right\}\left\{\lambda^{\prime \prime}\right\}$ and the empty boxes mean zero. The Schur functions in the upper edge correspond to the products that appear in the left edge while the ones of the bottom edge correspond to the products that appear in the right edge.

Plethysms will be considered in the next subsection.

\section{II.2 Invariant Matrices and Plethysms}

Let $A \equiv\left(a_{i j}\right)$ be a nonsingular matrix of dimension $n \times n$ representing the linear inversible transformation $\mathcal{A}$ of one point $X$ in a point $X^{\prime}$ of a $n$-dimensional vector space. In terms of coordinates one has

$$
\left[X^{\prime}\right]=A[X]
$$

where $[X]=\left[x_{1}, x_{2}, \ldots, x_{n}\right]^{T}$ and $[X \quad \prime]=$ $\left[x_{1}^{\prime}, x_{2}^{\prime}, \ldots, x_{n}^{\prime}\right]^{T}$ are column vectors in the coordinates of $X$ and $X^{\prime}$. One can construct $\left(\begin{array}{l}\mathrm{n}+\mathrm{r}-1 \\ \mathrm{r}\end{array}\right)$ homogeneous products of degree $r$ in the coordinates $x_{i}$ of $X$ :

$$
x_{1}^{r_{1}} x_{2}^{r_{2}} \ldots x_{n}^{r_{n}}, \quad r_{i} \geq 0, \quad \sum_{i=1}^{n} r_{i}=r
$$

Each of those products transforms, under the transformation $\mathcal{A}$, into a linear combination of themselves. Defining a column vector $[X]^{r}$ whose components are those products enumerated in an arbitrary way, its transformed under $\mathcal{A}$ can be written as

$$
\left([X]^{r}\right)^{\prime}=A^{[r]}[X]^{r},
$$

what defines a matrix $A^{[r]}$ called the $r$-induced matrix of the matrix $A$. The elements of $A^{[r]}$ are clearly homogeneus polynomials of degree $r$ in the components of A.

Making the same operation with a nonsingular $n \times n$ matrix $B$ one obtains $B^{[r]}$. If $C=A B$, then it can be proved that

$$
C^{[r]}=(A B)^{[r]}=A^{[r]} B^{[r]}
$$

what means that the set of matrices $\mathcal{D}^{[r]}(A) \equiv A^{[r]}$ is a $\left(\begin{array}{l}\mathrm{n}+\mathrm{r}-1 \\ \mathrm{r}\end{array}\right)$-dimensional representation of the group $G L(n)$ of the nonsingular matrices of dimension $n \times n$. As example, one has for $r=2$ :

$$
x_{i}^{\prime} x_{j}^{\prime}=\sum_{k, l} a_{i k} a_{j l} x_{k} x_{l}=\sum_{k}\left(a_{i k} a_{j k}\right) x_{k}^{2}+\sum_{k<l}\left(a_{i k} a_{j l}+a_{i l} a_{j k} x_{k} x_{l}\right.
$$

what implies in

$$
A_{(i j)(k k)}^{[2]}=a_{i k} a_{j k}, \quad i \leq j ; \quad A_{(i j)(k l)}^{[2]}=a_{i k} a_{j l}+a_{i l} a_{j k}, \quad i \leq j, k<l .
$$

For $n=2$ and choosing the ordering $\left(x_{1}^{2}, x_{1} x_{2}, x_{2}^{2}\right)$ one has

$$
A^{[2]}=\left(\begin{array}{ccc}
a_{11}^{2} & 2 a_{11} a_{12} & a_{12}^{2} \\
a_{11} a_{21} & \left(a_{11} a_{22}+a_{12} a_{21}\right) & a_{12} a_{22} \\
a_{21}^{2} & 2 a_{21} a_{22} & a_{22}^{2}
\end{array}\right), \quad n=2 .
$$

It should be noticed that the trace of $A^{[2]}$ is

$$
\operatorname{tr}\left(A^{[2]}\right)=a_{11}^{2}+a_{22}^{2}+a_{11} a_{22}+a_{12} a_{21}=\alpha_{1}^{2}+\alpha_{2}^{2}+\alpha_{1} \alpha_{2}=h_{2}=\{2\}(\alpha),
$$

where $\{2\}(\alpha)$ is the Schur function $\{2\}$ computed with the eigenvalues of $A$.

For any other nonsingular $2 \times 2$ matrices $B, C, D, \ldots$ one can construct their second induced matrices $B^{[2]}, C^{[2]}, D^{[2]}, \ldots$ as the matrices whose components are the same polynomials of degree 2 of $A$ in which now the variables are the corresponding elements of matrices $B, C, D, \ldots$. It is immediate to prove, by hand calculation, that

$$
A^{[2]} B^{[2]}=(A B)^{[2]}, \quad \forall A, B, \quad 2 \times 2
$$

what agrees with Eq.(17). Let us take now $r(<n)$ copies of the vector $X$ and build the $\left(\begin{array}{c}\mathrm{n} \\ \mathrm{r}\end{array}\right)$ determinants

$$
d_{\left(e_{1} e_{2} \ldots e_{p}\right)}=\left|\begin{array}{cccc}
x_{e_{1}}^{(1)} & x_{e_{1}}^{(2)} & \ldots & x_{e_{1}}^{(r)} \\
x_{e_{2}}^{(1)} & x_{e_{2}}^{(2)} & \ldots & x_{e_{2}}^{(r)} \\
\ldots & \ldots & \ldots & \ldots \\
x_{e_{r}}^{(1)} & x_{e_{r}}^{(2)} & \ldots & x_{e_{r}}^{(r)}
\end{array}\right|, \quad 1 \leq e_{1}<e_{2}<\ldots<e_{r} \leq n .
$$


If each $X^{(s)}$ is transformed by the same transformation $\mathcal{A}$, these determinants will transform linearly among themselves by the relation

$$
[d]^{\prime}=A^{(r)}[d]
$$

where $[d]$ is the column vector whose components are the $d_{\left(e_{1} e_{2} \ldots e_{r}\right)}$ enumerated in an arbitrary way. Relation (21) defines the matrix $A^{[r]}$, the $r$ th compound matrix of $A$. It follows from this definition that the matrix elements of $A^{(r)}$ are the minors of degree $r$ of the original matrix $A$.

If $C=A B$, then one can also demonstrate that

$$
C^{(r)}=(A B)^{(r)}=A^{(r)} B^{(r)} .
$$

As example, for $r=2$ one has

$$
\begin{aligned}
d_{e_{i} e_{j}}{ }^{\prime} & =\left|\begin{array}{cc}
x_{e_{i}}^{(1)} & x_{e_{i}}^{(2)} \\
x_{e_{j}}^{(1)} & x_{e_{j}}^{(2)}
\end{array}\right|=\left|\begin{array}{cc}
\sum_{k} a_{e_{i} k} x_{k}^{(1)} & \sum_{k} a_{e_{i} k} x_{k}^{(2)} \\
\sum_{l} a_{e_{j} l} x_{l}^{(1)} & \sum_{l} a_{e_{j} l} x_{l}^{(2)}
\end{array}\right|= \\
& =\sum_{k, l}\left|\begin{array}{cc}
a_{e_{i}} x_{k}^{(1)} & a_{e_{i} k} x_{k}^{(2)} \\
a_{e_{j} l} x_{l}^{(1)} & a_{e_{j} l} x_{l}^{(2)}
\end{array}\right|=\sum_{k<l}\left|\begin{array}{cc}
a_{i k} & a_{j k} \\
a_{i l} & a_{j l}
\end{array}\right| d_{e_{k} e_{l}} .
\end{aligned}
$$

Then, for $n=2$ one has

$$
A^{(2)}=a_{11} a_{22}-a_{12} a_{21}=\operatorname{det}(A), \quad n=2 .
$$

For $n=3$, with the ordering $d_{12}, d_{13}, d_{23}$ one has

$$
A^{(2)}=\left(\begin{array}{lll}
a_{11} a_{22}-a_{12} a_{21} & a_{11} a_{23}-a_{13} a_{21} & a_{11} a_{23}-a_{13} a_{23} \\
a_{11} a_{32}-a_{12} a_{31} & a_{11} a_{33}-a_{13} a_{31} & a_{12} a_{33}-a_{13} a_{32} \\
a_{21} a_{32}-a_{22} a_{31} & a_{21} a_{33}-a_{23} a_{31} & a_{22} a_{33}-a_{23} a_{32}
\end{array}\right), \quad n=3 .
$$

If $C=A B$ then, again by hand calculation, one can prove that

$$
C^{(2)}=(A B)^{(2)}=A^{(2)} B^{(2)} .
$$

The $r$-induced and $r$-compound matrices are particular cases of invariant matrices whose definition are given in the following.

Let $T(A)$ be a matrix whose elements are polynomials $t_{i j}$ having as variables the matrix elements of $A$. Let $T(B)$ be a matrix built with the same polynomials of $T(A)$ now in the matrix elements of $B$. If

$$
T(A) T(B)=T(A B)
$$

for any nonsingular $n \times n$ matrices $A, B$ then the matrix $T(A)$ is called a invariant matrix of $A$.

It follows from (26) that, once the set of polynomials $t_{i j}$ is fixed, the set of matrices $\mathcal{D}^{T}(A) \equiv T(A)$ is a representation of $G L(n)$.

A trivial case is the 1-induced matrix. In this case one has $t_{i j}=a_{i j}$ and $A^{[1]} \equiv A$. As it is known, the trace of $A$ is the sum of its eigenvalues $\alpha_{i}$, that is,

$$
\operatorname{tr}(A)=\sum_{i} \alpha_{i}=a_{1}(\alpha)=\{1\}(\alpha)
$$

where $\{1\}(\alpha)$ is the Schur function $\{1\}$ calculated with the eigenvalues of $A$. This will be, by definition, $\mathcal{D}^{[1]}$.

Since the Kronecker product of two representations is also a representation and the product of two polynomials is also a polynomial, it follows that the kronecker product of two invariant matrices is also an invariant matrix.

Littlewood[1] showed that if the product of two invariant matrices is reducible, then it will be totally reducible. This allows us to obtain new irreps by taking multiple Kronecker products of the irrep $\mathcal{D}^{[1]}$ and reducting them by similarity transformations.

As a sake of example, let us take $n=p=2$. In this case one has $A^{[2]}$ and $A^{(2)}$ given by (19) and (23). Writing $A \times A$ in the form

$$
A \times A=\left(\begin{array}{cccc}
a_{11} a_{11} & a_{11} a_{12} & a_{12} a_{11} & a_{12} a_{12} \\
a_{11} a_{21} & a_{11} a_{22} & a_{12} a_{21} & a_{12} a_{22} \\
a_{21} a_{11} & a_{21} a_{12} & a_{22} a_{11} & a_{22} a_{12} \\
a_{21} a_{21} & a_{21} a_{22} & a_{22} a_{21} & a_{22} a_{22}
\end{array}\right)
$$

one verifies that 


$$
\begin{aligned}
M^{-1}(A \times A) M & =\left(\begin{array}{cccc}
a_{11}^{2} & 2 a_{11} a_{12} & a_{12}^{2} & 0 \\
a_{11} a_{21} & a_{11} a_{22}+a_{12} a_{21} & a_{12} a_{22} & 0 \\
a_{21}^{2} & 2 a_{21} a_{22} & a_{22}^{2} & 0 \\
0 & 0 & 0 & a_{11} a_{22}-a_{12} a_{21}
\end{array}\right) \\
& =A^{[2]}+A^{(2)}
\end{aligned}
$$

with

$$
M=\left(\begin{array}{cccc}
1 & 0 & 0 & 0 \\
0 & 1 & 0 & -1 \\
0 & 1 & 0 & 1 \\
0 & 0 & 1 & 0
\end{array}\right)
$$

This shows that, for $n=2, A^{[1]} \times A^{[1]}$ reduces into $A^{[2]}+A^{(2)}$.

For $r=2$ and any $n$ one has $\left(\begin{array}{c}\mathrm{n}+2-1 \\ 2\end{array}\right)+\left(\begin{array}{c}\mathrm{n} \\ 2\end{array}\right)=$ $n^{2}$. Since $\operatorname{tr}(A \times A)=(\operatorname{tr}(A))^{2}$ this drives us to suppose that

$$
A^{[1]} \times A^{[1]}=A^{[2]}+A^{(2)}
$$

for any $n$, what turns out to be true since the Kronecker product of two matrices can be decomposed in a symmetric and an antisymmetric part relative to the components of the first and second factor matrices.

An analogous analysis can be done for the $r$ Kronecker product $M \times M \times \ldots \times M$. Based in this analysis, Schur[4] demonstrated that "If $A$ is an $n \times n$ matrix, there are as many irreducible invariant matrices of $A$ of degree $r$ as are the partitions of $r$ with no more than $n$ parts and the trace of them are the Schur functions of degree $r$ in the eigenvalues of $A . "$

Those irreducible matrices can then be labeled by the possible partitions of $r$ in no more than $n$ parts. In this notation one writes $A^{(r)} \equiv A^{\left[1^{r}\right]}$. Clearly, for $r>n$ only the irreps with up to $n$ rows are realizable.

The details of the effective construction of an irreducible invariant matrix corresponding to a given partition can be found in Refs[8], [14].

Using the components of the Schur functions to label the $G L(n)$ irreps, the above result shows that

" The $G L(n)$ irreps can be labeled by $\left[m_{1 n}, m_{2 n}, \ldots, m_{n n}\right]$ where the $m_{i n}$ satisfy the relations $m_{\text {in }} \geq m_{i+1 n}, 1 \leq i \leq n$ and the trace of each element $A$ of $G L(n)$ is the Schur function $\left\{m_{1 n}, m_{2 n}, \ldots, m_{n n}\right\}$ evaluated with the eigenvalues of $A$."
Since the $G L(n)$ irreps do not reduce under the restriction $G L(n) \rightarrow U(n)$ the same result holds for $U(n)$.

Let $T^{\prime}$ and $T^{\prime \prime}$ be two representations of $G L(n)$ by invariant matrices. From (26) it follows that

$$
T^{\prime \prime}\left(T^{\prime}(A B)\right)=\left(T ^ { \prime \prime } ( T ^ { \prime } ( A ) ) \left(T^{\prime \prime}\left(T^{\prime}(B)\right)\right.\right. \text {. }
$$

Since the product of two polynomials is also a polynomial, it follows that any invariant matrix of an invariant matrix is also an invariant matrix of the original matrix and therefore can be expressed as a direct sum of irreducible invariant matrices. This allows us to write

$$
\left[A^{[\mu]}\right]^{[\nu]}=\sum_{\lambda} \kappa_{\lambda \mu \nu} A^{[\lambda]} .
$$

Let us denote by $r_{\mu}, r_{\nu}$ e $r_{\lambda}$ the degrees of $[\mu],[\nu]$ and $[\lambda]$. Since $A^{[\mu]}$ is obtained by the reduction of the $r_{\mu^{-}}$ ple Kronecker product of $A$ and, by its turn, $\left[A^{[\mu]}\right]^{[\nu]}$ is obtained reducing the $r_{\nu}$-ple Kronecker product of $A^{[\mu]}$ it follows that the irreps that appear in the RHS of (29) have degree $r_{\lambda}=r_{\mu} r_{\nu}$.

Since the trace of an irreducible invariant matrix is equal to the Schur function of the correponding partition, Eq.(29) allows us to stablish a correspondence $\{\mu\} \otimes\{\nu\}$ between two Schur functions $\{\lambda\}$ of degrees $r_{\mu}$ and $r_{\nu}$ with Schur functions $\{\lambda\}$ of degree $r_{\mu} r_{\nu}$ given by

$$
\{\mu\} \otimes\{\nu\}=\sum_{\lambda} \kappa_{\lambda \mu \nu}\{\lambda\},
$$

where the coefficients $\kappa_{\lambda \mu \nu}$ are given by (29).

This correspondence was descovered by Littlewood[14] and later was named plethysm.

The plethysm operation has the properties[3],[2],

[14]

$$
\{\lambda\} \otimes(\{\mu\} \otimes\{\nu\})=(\{\lambda\} \otimes\{\mu\}) \otimes\{\nu\},
$$




$$
\begin{aligned}
\{\lambda\} \otimes(\{\mu\} \pm\{\nu\})= & \{\lambda\} \otimes\{\mu\} \pm\{\lambda\} \otimes\{\nu\}, \\
(\{\lambda\}+\{\mu\}) \otimes\{\nu\}= & \sum_{\lambda^{\prime} \lambda^{\prime \prime}} \alpha\left(\left\{\lambda^{\prime}\right\}\left\{\lambda^{\prime \prime}\right\} \rightarrow\{\nu\}\right)\left(\{\lambda\} \otimes\left\{\lambda^{\prime}\right\}\right) \cdot \\
& \left(\{\mu\} \otimes\left\{\left\{\lambda^{\prime \prime}\right\}\right) .\right. \\
(\{\lambda\}-\{\mu\}) \otimes\{\nu\}= & \sum_{\lambda^{\prime} \lambda^{\prime \prime}}(-)^{r^{\prime \prime}} \alpha\left(\left\{\lambda^{\prime}\right\}\left\{\lambda^{\prime \prime}\right\} \rightarrow\{\nu\}\right)\left(\{\lambda\} \otimes\left\{\lambda^{\prime}\right\}\right) . \\
& \cdot\left(\{\mu\} \otimes\left\{\tilde{\lambda}^{\prime \prime}\right\}\right) \\
\{\lambda\} \otimes(\{\mu\}\{\nu\})= & (\{\lambda\} \otimes\{\mu\})(\{\lambda\} \otimes\{\nu\}) \\
(\{\lambda\}\{\mu\}) \otimes\{\nu\}= & \sum_{\lambda^{\prime} \lambda^{\prime \prime}} \alpha\left(\left\{\lambda^{\prime}\right\} \times\left\{\lambda^{\prime \prime}\right\} \rightarrow\{\nu\}\right)\left(\{\lambda\} \otimes\left\{\lambda^{\prime}\right\}\right) . \\
& \cdot\left(\{\mu\} \otimes\left\{\lambda^{\prime \prime}\right\}\right) \\
(\{\lambda\} \otimes\{\mu\})^{T}= & \begin{cases}\{\widetilde{\lambda}\} \otimes\{\mu\}, \quad \text { for } r_{\lambda} \text { even, } \\
\{\widetilde{\lambda}\} \otimes\{\widetilde{\mu}\}, \quad \text { for } r_{\lambda} \text { odd. }\end{cases}
\end{aligned}
$$

The sum in Eq.(33) includes the cases $\left\{\lambda^{\prime}\right\}=\{0\} \equiv 1,\left\{\lambda^{\prime \prime}\right\}=\{\nu\}$ and $\left\{\lambda^{\prime}\right\}=\{\nu\},\left\{\lambda^{\prime \prime}\right\}=\{0\} \equiv 1$. Symilarly in $\operatorname{Eqs}(34)$ and (36). Also, $r^{\prime \prime}$ and $r_{\lambda}$ are the degrees of $\left\{\lambda^{\prime \prime}\right\}$ and $\{\lambda\}$.

In Eq.(37) we used the notation

$$
\left[\sum_{i} a_{i}\left\{\lambda_{(i)}\right\}\right]^{T}=\sum_{i} a_{i}\left\{\tilde{\lambda}_{(i)}\right\}
$$

where $a_{i}$ are numerical factors, $\left\{\lambda_{(i)}\right\}$ Schur functions and $\left\{\widetilde{\lambda}_{(i)}\right\}$ their conjugate.

There is also a dimension formula [2],[3],[33]

$d_{\{\lambda\} \otimes\{\mu\}} \equiv \sum_{\nu} \alpha(\{\lambda\} \otimes\{\mu\} \rightarrow\{\nu\}) d_{[\nu]}=\frac{(r s) !}{(r !)^{s} s !}\left(d_{[\lambda]}\right)^{s} d_{[\mu]}$,

where $r$ and $s$ are the degrees of $[\lambda]$ and $[\mu]$ and $d_{[\sigma]}$ is the dimension of the irrep $[\sigma]$ of $S$ (degree of $[\sigma]$ ) .

Up to now we have seen plethysms of Schur functions of $G L(n)$. One can obtain plethysms of continuous subgroups $G$ of $G L(n)$ simply by expressing the irreducible characters of $G$ in terms of Schur functions and then computing the plethysm of Schur functions. If necessary, the result can be re-expressed in terms of the irreducible characters of $G$. This will be done when we will consider the applications.

In the case of $G=S(n)$, a finite subgroup of $G L(n)$ of great importance in the study of systems of identical particles, this process involves the concept of inner plethysm which we shall consider in the following.

As we have seen previously, the inner product $\{\lambda\} \times\{\mu\}$ of two Schur functions of the same degree $r$ corresponds to the Kronecker product of two irreps $[\lambda]$ and $[\mu]$ of $S(r)$. The case $\{\lambda\} \times\{\lambda\}$ can be analysed in its symmetric and antisymmetric constituents. The Kronecker product $M \times M$ of a matrix $M$ by itself decomposes as

$$
M \times M \doteq M^{[2]}+M^{\left[1^{2}\right]} .
$$

Taking by $M$ the matrix that represents a given permutation of $S(n)$ in the irrep [ $\lambda]$, correpondently, the expression $\{\lambda\} \times\{\lambda\}$ can be analized in two parts, denoted by $\{\lambda\} \odot\{2\}$ and $\{\lambda\} \odot\left\{1^{2}\right\}$. The operation denoted by the symbol $\odot$ is called inner plethysm[13] and will be defined in the following.

Let $M_{i}$ be a matrix of a generic permutation $P_{i}$ of $S(n)$ and $\chi^{[\lambda]}\left(P_{i}\right)$ its trace. Then the invariant matrix of $M_{i}$ associated to the partition [ $\left.\mu\right]$, that is, $M_{i}^{[\mu]}$, also represents the element $P_{i}$ and its trace is a compound character, say,

$$
\operatorname{tr}\left(M_{i}^{[\mu]}\right)=\sum_{\nu} G_{\lambda \mu \nu} \chi^{[\nu]}\left(P_{i}\right)
$$

One defines $\{\lambda\} \odot\{\mu\}$, the inner plethysm of partitions $\{\lambda\}$ and $\{\mu\}$ of degrees $n$ and $m$, as a linear combination of partitions $\{\nu\}$ of degree $n$ with coefficients given by Eq.(41), that is,

$$
\{\lambda\} \odot\{\mu\}=\sum_{\nu} G_{\lambda \mu \nu}\{\nu\}
$$


Notice that although the degree of partitions $[\lambda]$ and $[\nu]$ is $n$, there is no restriction concerning the degree of partition $[\mu]$.

The inner plethysms have properties analogous to those of plethysms, roughly changing outer products of Schur functions by inner products, that is,

$$
\begin{aligned}
(\{\lambda\} \odot\{\mu\}) \odot\{\nu\} & =\{\lambda\} \odot(\{\mu\} \odot\{\nu\}), \\
\{\lambda\} \odot(\{\mu\}+\{\nu\}) & =\{\lambda\} \odot\{\mu\}+\{\lambda\} \odot\{\nu\}, \\
(\{\lambda\}+\{\mu\}) \odot\{\nu\} & =\sum_{\rho \sigma} \alpha(\{\rho\}\{\sigma\} \rightarrow\{\nu\})(\{\lambda\} \odot\{\rho\}) \times(\{\mu\} \odot\{\sigma\}), \\
\{\lambda\} \odot(\{\mu\}\{\nu\}) & =(\{\lambda\} \odot\{\mu\}) \times(\{\lambda\} \odot\{\nu\}), \\
(\{\lambda\}\{\mu\}) \odot\{\nu\} & =\sum_{\rho \sigma} \alpha(\{\rho\} \times\{\sigma\} \rightarrow\{\nu\})(\{\lambda\} \odot\{\rho\}) \times(\{\mu\} \odot\{\sigma\}) .
\end{aligned}
$$

\section{Plethysms Calculation}

In [3] one finds the following expression for calculation of plethysm:

$$
\left\{\lambda^{\prime}\right\} \otimes\left\{\lambda^{\prime \prime}\right\}=\sum_{\left(\rho^{\prime \prime}\right)} \frac{n^{\prime \prime}\left(\rho^{\prime \prime}\right)}{r^{\prime \prime} !} \chi^{\left[\lambda^{\prime \prime}\right]}\left(\rho^{\prime \prime}\right)\left(S_{1}^{\prime \prime}\right)^{\rho_{1}^{\prime \prime}}\left(S_{2}^{\prime \prime}\right)^{\rho_{2}^{\prime \prime}} \ldots\left(S_{n^{\prime \prime}}^{\prime \prime}\right)^{\rho_{n^{\prime \prime}}^{\prime \prime}}
$$

where

$r^{\prime}$ and $r^{\prime \prime}$ are the $\left\{\lambda^{\prime}\right\}$ and $\left\{\lambda^{\prime \prime}\right\}$ degrees, $\left(\rho^{\prime \prime}\right)=\left(1^{\rho_{1}^{\prime \prime}}, 2^{\rho_{2}^{\prime \prime}}, \ldots\right)$ are the $S\left(r^{\prime \prime}\right)$ classes and $n^{\prime \prime}\left(\rho^{\prime \prime}\right)$ their number of elements,

$$
S_{i}^{\prime \prime}=\sum_{[\lambda]_{i r^{\prime}}}\{\lambda\}_{i n^{\prime}} \sum_{\left(\rho^{\prime}\right)} \frac{n^{\prime}\left(\rho^{\prime}\right)}{r^{\prime} !} \chi^{\left[\lambda^{\prime}\right]}\left(\rho^{\prime}\right) \chi^{[\lambda]_{i r^{\prime}}}\left(\rho_{0}\left(\rho^{\prime}\right)\right)
$$

$\rho_{0}\left(\rho^{\prime}\right)$ is the $S\left(i r^{\prime}\right)$ class with $\rho_{1}^{\prime}$ cicles of length $1, \rho_{2}^{\prime}$ cicles of length 2 , etc.

The Schur functions $\left\{\lambda^{\prime \prime \prime}\right\}$, having degree $r^{\prime \prime \prime}=r^{\prime} \times r^{\prime \prime}$, which enter in the plethysm are obtained after reducing the outer products of Schur functions inside the $S_{i}^{\prime \prime}$. For small values of $r^{\prime \prime \prime}$ it is possible to construct plethysms tables with the same organization of the tables of outer products. One has, as an example, for $r^{\prime \prime \prime}=6$ the table below

\begin{tabular}{|l|l|l|l|l|l|l|l|l|l|}
\cline { 2 - 10 } \multicolumn{1}{c|}{} & $\{6\}$ & $\{51\}$ & $\{42\}$ & $\left\{41^{2}\right\}$ & $\left\{3^{2}\right\}$ & $\{321\}$ & $\left\{31^{3}\right\}$ & $\left\{2^{3}\right\}$ & \\
\hline$\{2\} \otimes\{3\}$ & 1 & & 1 & & & & & 1 & $\left\{1^{2}\right\} \otimes\{3\}$ \\
\hline$\{2\} \otimes\{21\}$ & & 1 & 1 & & & 1 & & & $\left\{1^{2}\right\} \otimes\{21\}$ \\
\hline$\{2\} \otimes\left\{1^{3}\right\}$ & & & & 1 & 1 & & & & $\left\{1^{2}\right\} \otimes\left\{1^{3}\right\}$ \\
\hline$\{3\} \otimes\{2\}$ & 1 & & 1 & & & & & & $\left\{1^{3}\right\} \otimes\left\{1^{2}\right\}$ \\
\hline$\{3\} \otimes\left\{1^{2}\right\}$ & & 1 & & & 1 & & & & $\left\{1^{3}\right\} \otimes\{2\}$ \\
\hline$\{21\} \otimes\{2\}$ & & & 1 & & & 1 & 1 & 1 & $\{21\} \otimes\left\{1^{2}\right\}$ \\
\hline
\end{tabular}

Table II: All plethysms that produce Schur functions of degree 6 .

In [3] one can find plethysms tables for $r^{\prime \prime \prime}$ up to 10. Ibrahin [15],[16] published tables with $r^{\prime \prime \prime}=12,14$ and 15 . Notice that such tables exist only for $r^{\prime \prime \prime}$ nonprime. Partial tables were published in [2] and [21].

For great values of $r^{\prime \prime \prime}$ the use of (44) is unpractical, then the need for alternative formulas. For some particular cases of $\left\{\lambda^{\prime}\right\}$ and $\left\{\lambda^{\prime \prime}\right\}$ there exist explicit (or almost) formulas easy to use. They are the following:

$$
\begin{aligned}
& \{\lambda\} \otimes\{1\}=\{1\} \otimes\{\lambda\}=\{\lambda\} \\
& \{\lambda\} \otimes\{0\}=\{0\}, \quad\{0\} \otimes\{\lambda\}=\delta_{\{\lambda\},\left\{r_{\lambda}\right\}}\{0\} \\
& \{r\} \otimes\{2\}=\sum_{i=0}^{[r / 2]}\{2 r-2 i, 2 i\}
\end{aligned}
$$




$$
\begin{aligned}
\{r\} \otimes\left\{1^{2}\right\} & =\sum_{i=1}^{[(r+1) / 2]}\{2 r-(2 i-1),(2 i-1)\} \\
\{2\} \otimes\{r\} & =\sum_{\lambda}\{\lambda\}_{\text {even }} ; \\
\left\{1^{2}\right\} \otimes\{r\} & =\sum_{\lambda}\left\{\widehat{\lambda\}_{\text {even }}} ;\right. \\
\left\{1^{r}\right\} \otimes\{2\} & =\left\{1^{2 r}\right\}+\sum_{i=1}^{[r / 2]}\left\{2^{2 i}, 1^{2 r-4 i}\right\}, r \text { even, } \\
\left\{1^{r}\right\} \otimes\left\{1^{2}\right\} & =\left\{1^{2 r}\right\}+\sum_{i=1}^{[r / 2]}\left\{2^{2 i}, 1^{2 r-4 i}\right\}, \text { r odd } \\
\left\{1^{r}\right\} \otimes\left\{1^{2}\right\} & =\sum_{i=1}^{[(r+1) / 2]}\left\{2^{2 i-1}, 1^{2 r-2(2 i-1)}\right\}, r \text { even } \\
\left\{1^{r}\right\} \otimes\{2\} & =\sum_{i=1}^{[(r+1) / 2]}\left\{2^{2 i-1}, 1^{2 r-2(2 i-1)}\right\}, r \text { odd }
\end{aligned}
$$

Eq.(46) follows from plethysms definition while Eq.(47) is set for consistency. In Eq.(50), $\{\lambda\}_{\text {even }}$ means partition of $2 r$ with all lines even. Eqs.(54) and (55) follow from Eqs.(48) and (49) by conjugation[ see Eq.(37).].

In [21] there are formulas for the calculation of plethysms $\{\lambda\} \otimes\{\mu\}$ when both Schur functions are symmetric or/and antisymmetric. To explain them we need the following definition: a $k$-border strip of a given Young diagram $[\lambda]$ is a sequence of $k$ squares in which the first of them is the last one of the first line of $[\lambda]$ and the next square to a given one is the one below it, if it exists, or the one to its left, otherwise.

For example, the 3 -border strips of $\left[41^{2}\right],[321]$ and $\left[2^{2} 1^{2}\right]$ are the squares with the symbol $\mathbf{\square}$ in the figures below, respectively:
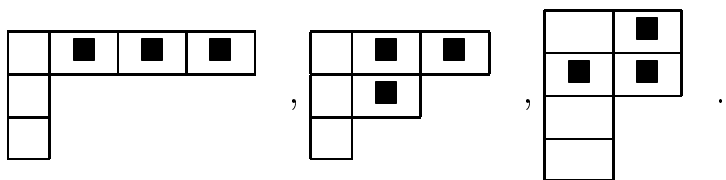

When $\{\lambda\}$ and $\{\mu\}$ are both symmetric, one has

$$
\{n\} \otimes\{m\}=\frac{1}{m} \sum_{k=1}^{m}\{n\}\left(x^{k}\right)(\{n\} \otimes\{m-k\}), \quad m \geq 1,
$$

with

$$
\{n\}\left(x^{k}\right)=\sum_{\nu} C_{n, k, \nu}\{\nu\} .
$$

In (57) the $\{\nu\}$ are all Schur functions of degree $n k$. The coefficients $C_{n, k, \nu}$ are obtained from the Young diagram associated to $\{\nu\}$ removing, in sequence, $n k$-border strips. If in all steps the resulting diagram be regular then

$$
C_{n, k, v}=(-)^{l}
$$

with $l=$ (number of lines in the $k$-border strips) $-n$. If in any step the resulting diagram be not regular, then $C_{n, k, v}=0$. As example, from the figures above one has

$$
C_{2,3,\left\{41^{2}\right\}}=(-)^{4-2}=1 ; \quad C_{2,3,\{321\}}=(-)^{5-2}=-1 ; \quad C_{2,3,\left\{2^{2} 1^{2}\right\}}=0 .
$$

The coefficient $C_{2,3,\left\{2^{2} 1^{2}\right\}}$ is zero because after removing the first 3-border strip the resulting diagram corresponds to $\left\{\nu^{\prime}\right\}=\left\{1,0,1^{2}\right\}$, being therefore not regular. 
Eq.(56) allows one to relate the plethysm of two symmetric Schur functions with the plethysms of symmetric Schur functions of smaller degrees. In this way, using $\{n\} \otimes\{1\} \equiv\{n\}$ as starting point one computes all the plethysms of type $\{n\} \otimes\{m\}$.

Using the result

$$
\left\{1^{m}\right\}=(-)^{m+1}\{m\}+\sum_{k=1}^{m-1}(-)^{k+1}\{k\}\left\{1^{m-k}\right\}, \quad m \geq 1
$$

and Eqs.(32) one obtains

$$
\begin{array}{r}
\{n\} \otimes\left\{1^{m}\right\}=(-)^{m+1}\{n\} \otimes\{m\}+\sum_{k=1}^{m-1}(-)^{k+1}(\{n\} \otimes\{k\})\left(\{n\} \otimes\left\{1^{m-k}\right\}\right) \\
\left\{1^{n}\right\} \otimes\left\{1^{m}\right\}=(-)^{m+1}\left\{1^{n}\right\} \otimes\{m\}+\sum_{k=1}^{m-1}(-)^{k+1}\left(\left\{1^{n}\right\} \otimes\{k\}\right)\left(\left\{1^{n}\right\} \otimes\left\{1^{m-k}\right\}\right)
\end{array}
$$

For $\left\{1^{n}\right\} \otimes\{m\}$ one uses

$$
\left\{1^{n}\right\} \otimes\{m\}= \begin{cases}{[\{n\} \otimes\{m\}]^{T},} & \text { for } n \text { even, } \\ {\left[\{n\} \otimes\left\{1^{m}\right\}\right]^{T},} & \text { for } n \text { odd. }\end{cases}
$$

In this way, using (56) and (60)-(62) one computes plethysms with both Schur functions symmetric and/or antisymmetric.

The cases in which $\{\lambda\}\{\mu\}$ has a closed and simple expression allow us to compute, using (32) and (35) relations also closed for the corresponding plethysms. In this way, from

$$
\{p\}\{q\}=\sum_{i=0}^{q}\{p+q-i, i\}, \quad p \geq q
$$

one obtains $\{p, q\}=\{p\}\{q\}-\{p+1, q-1\}$. From this equation, using (32), (35) and iterating one obtains

$$
\{\lambda\} \otimes\{p, q\}=\sum_{i=0}^{q}(-)^{i}(\{\lambda\} \otimes\{p+i\})(\{\lambda\} \otimes\{q-i\}),
$$

what allows us to express a plethysm with a two-row Schur function at right in terms of plethysms with symmetric Schur functions at right.

In an analogous way, from

$$
\left\{1^{p}\right\}\left\{1^{q}\right\}=\sum_{i=0}^{q}\left\{2^{q-i}, 1^{p-q+2 i}\right\}, \quad p \geq q,
$$

one obtains

$$
\{\lambda\} \otimes\left\{2^{q}, 1^{p-q}\right\}=\left(\{\lambda\} \otimes\left\{1^{p}\right\}\right)\left(\{\lambda\} \otimes\left\{1^{q}\right\}-\{\lambda\} \otimes\left\{1^{p+1}\right\}\right)\left(\{\lambda\} \otimes\left\{1^{q-1}\right\},\right.
$$

what express a plethysm with a two-column Schur function at right in terms of plethysms with one-column Schur functions at right.

An analogous procedure is used to compute plethysms with Schur functions at left with mixed symmetry using (33), (35) and (36). Due to the more complex nature of these equations as compared with that for Schur functions to the right the calculation is more complicated.

When the degree $n$ of the Schur function at left is a compound integer one can use the tables of plethysms with final degree $n$ to obtain relations in general simpler than the one here exposed. As example, from $\{2\} \otimes\left\{1^{2}\right\}=\{31\}$ one obtains

$$
\{31\} \otimes\{\mu\}=\left(\{2\} \otimes\left\{1^{2}\right\}\right) \otimes\{\mu\}=\{2\} \otimes\left(\left\{1^{2}\right\} \otimes\{\mu\}\right)
$$

and also $\left\{21^{2}\right\} \otimes\{\mu\}$ by conjugation. 
A very common situation which arrises in applications is when one needs to compute plethysms of a same Schur function by many (sometimes all) Schur functions of a given degree to the right.[ This is the case of the application that we will make in Section 4.] For such cases we present the following algorithm that allows to compute, in a build up way, all plethysms $\{\lambda\} \otimes\{\mu\}_{m}$ with $\{\lambda\}$ a fixed Schur function and $\{\mu\}_{m}$ all Schur functions of degree $m$, once the plethysms $\{\lambda\} \otimes\{m\}$ and $\{\lambda\} \otimes\{\mu\}_{m^{\prime}}$, with $m^{\prime}<m$ have already been computed :

1) Find all partitions of $m$ and order them in descending order of their components read from left to right;

2) For each partition $\{\mu\}=\left\{\mu_{1}, \mu_{2}, \ldots, \mu_{t}, 0, \ldots, 0\right\}$ perform the outer product

$\left\{\mu_{1}, \mu_{2}, \ldots, \mu_{t-1}\right\}\left\{\mu_{t}\right\}$, order the irreps in the reduction as in item (1), then use Eqs.(32) to obtain the equation

$$
\begin{aligned}
\{\lambda\} \otimes\{\mu\}= & \left(\{\lambda\} \otimes\left\{\mu_{1}, \mu_{2}, \ldots, \mu_{t-1}\right\}\right)\left(\{\lambda\} \otimes\left\{\mu_{t}\right\}\right)- \\
& \sum_{\left\{\mu^{\prime}\right\} \prec\{\mu\}} \alpha\left(\left\{\mu_{1}, \mu_{2}, \ldots, \mu_{t-1}\right\}\left\{\mu_{t}\right\} \rightarrow\left\{\mu^{\prime}\right\}\right)\{\lambda\} \otimes\left\{\mu^{\prime}\right\}
\end{aligned}
$$

where the symbol $\prec$ means preceding, following the ordering in item 1).

Since $\left\{\mu_{1}, \mu_{2}, \ldots, \mu_{t-1}\right\}$ and $\left\{\mu_{t}\right\}$ have smaller degree than $\{\mu\}$, the plethysms $\{\lambda\} \otimes\left\{\mu_{1}, \mu_{2}, \ldots, \mu_{t-1}\right\}$ and $\{\lambda\} \otimes\left\{\mu_{t}\right\}$ have already been computed in the induction process. On the other hand, the plethysms $\{\lambda\} \otimes\left\{\mu^{\prime}\right\}$ also have been computed since $\left\{\mu^{\prime}\right\}$ precedes $\{\mu\}$.

As an example of the procedure suggested above, we list below the sequence of equations [obtained from Table I] that allows us to compute $\{\lambda\} \otimes\{\mu\}$ for all Schur functions $\{\mu\}$ with degree 5 , once $\{\lambda\} \otimes\{5\}$ and the plethysms with total degree less than $5 r_{\lambda}$ be known.

$$
\begin{aligned}
\{\lambda\} \otimes\{5\} & =\text { input, } \\
\{\lambda\} \otimes\{41\} & =(\{\lambda\} \otimes\{4\})\{\lambda\}-\{\lambda\} \otimes\{5\}, \\
\{\lambda\} \otimes\{32\} & =(\{\lambda\} \otimes\{3\})(\{\lambda\} \otimes\{2\})-\{\lambda\} \otimes\{5\}-\{\lambda\} \otimes\{41\}, \\
\{\lambda\} \otimes\left\{31^{2}\right\} & =(\{\lambda\} \otimes\{31\})\{\lambda\}-\{\lambda\} \otimes\{41\}-\{\lambda\} \otimes\{32\}, \\
\{\lambda\} \otimes\left\{2^{2} 1\right\} & =\left(\{\lambda\} \otimes\left\{2^{2}\right\}\right)\{\lambda\}-\{\lambda\} \otimes\{32\}, \\
\{\lambda\} \otimes\left\{21^{3}\right\} & =\left(\{\lambda\} \otimes\left\{1^{2}\right\}(\{\lambda\} \otimes\{2\})-\{\lambda\} \otimes\left\{31^{2}\right\},\right. \\
\{\lambda\} \otimes\left\{1^{5}\right\} & =\left(\{\lambda\} \otimes\left\{1^{3}\right\}\right)\left(\{\lambda\} \otimes\left\{1^{2}\right\}-\{\lambda\} \otimes\left\{21^{3}\right\}-\{\lambda\} \otimes\left\{2^{2} 1\right\} .\right.
\end{aligned}
$$

Since $\{n\} \otimes\{5\}$ one obtains from (56), we use it to obtain all the plethysms $\{n\} \otimes\{\mu\}$ of degree $n m$. For security sake, we recommend to make a dimension test using (39) for all new plethysm obtained.

\section{Applications}

In applications of group theory to problems of nuclear and atomic spectroscopy we are frequently led to consider the decomposition of characters of irreps of a group that acts over an $n$-dimensional vector space into those of one of its subgroup. The subgroup can also act over a $n$-dimensional vector space but in a restricted way. It can also be the direct product of two groups that act over $n_{1}$ - and $n_{2}$ - dimensional vector subspaces of the original space. As example, $S p(4 \ell+2) \rightarrow$ $S U(2) \times R(2 \ell+1), R(2 \ell+1) \rightarrow R(3), R(6) \rightarrow R(5)$ and $G L(2) \rightarrow R(3)$ have many applications in the theory of complex spectra [ We will use the notation $U$ for unitary, $O$ for orthogonal, $R=O^{+}=S O$ for unimodular orthogonal or rotation, $S p$ for symplectic and $G L$ for general linear groups.]. The algebra of plethysms give 
a simple and complete solution to this problem.

This solution is based in the theorem[2]

If under the restriction $G \rightarrow H$ the character $f 1\}$ of group $G$ decomposes as

$$
f 1\}=+\alpha+++\beta++\ldots++\omega+
$$

then the character $千 \lambda+$ of $G$ decomposes into the characters $+\rho \rightarrow$ of $H$ according to the characters contained in the plethysm

$$
[+\alpha+++\beta++\ldots++\omega+] \otimes f \lambda\} .
$$

This plethysm can be obtained expressing the characters of $G$ and $H$ in terms of characters of $G L(n)$, computing the resulting plethysms of $G L(n)$ characters and re-expressing the result in terms of characters of $H$ in order to obtain the final result.

Using the association irrep $\leftrightarrow$ character this theorem gives us the coefficients of the reduction of the irrep $\{\lambda\}$ of $G$ in the direct sum of irreps $(\rho)$ of $H$.

Let us consider now some illustrative examples of the appplications of the theorem. To this end we list below the decomposition of the character $f 1+$ of $G$ in terms of characters[2] $+\rho \rightarrow$ of $H$ :

$$
\begin{array}{lll}
\mathrm{U}(\mathrm{n}) & \rightarrow \mathrm{U}(\mathrm{n}-1): & \{1\}=\{1\}+\{0\} ; \\
\mathrm{U}(4 \ell+2) & \rightarrow \mathrm{SU}(2) \times O^{+}(2 \ell+1): & \{1\}=\left[\frac{1}{2}\right]^{\prime}(1) ; \\
\mathrm{O}^{+}(8 \ell+4) & \rightarrow \mathrm{SU}(2) \times S p(4 \ell+2): & (1)=\left[\frac{1}{2}\right]^{\prime}<1>; \\
\mathrm{Sp}(4 \ell+2) & \rightarrow \mathrm{SU}(2) \times R(2 \ell+1): & <1>\left[\frac{1}{2}\right]^{\prime}(1) ; \\
\mathrm{O}^{+}(2 \ell+1) & \rightarrow \mathrm{O}^{+}(3): & (1)=(\ell) ; \\
\mathrm{O}^{+}(6) & \rightarrow \mathrm{O}^{+}(5): & (1)=(1)+(0) ; \\
\mathrm{GL}(2) & \rightarrow \mathrm{O}^{+}(3): & \{1\}=(3) ; \\
\mathrm{U}(\mathrm{n}) & \rightarrow \mathrm{O}^{(\mathrm{n}):} & \{1\}=(1) ; \\
\mathrm{U}(\mathrm{s}) & \rightarrow \mathrm{U}_{S}(3) \oslash \mathrm{U}_{T}(3): & \{1\}=\{1\}_{S}\{0\}_{T}+\{0\}_{S}\{1\}_{T} ; \\
\mathrm{U}(\mathrm{nm}) & \rightarrow \mathrm{U}(\mathrm{n}) \times U(m) & \{1\}=\{1\}^{\prime}\{1\}^{\prime \prime} .
\end{array}
$$

By the above theorem and the first line of $(71)$, in order to obtain the $U(n-1)$ irreps contained in the $U(n)$ irrep $\{\lambda\}$ we must evaluate the plethysm

$$
\begin{aligned}
(\{1\}+\{0\}) \otimes\{\lambda\} & =\sum_{\lambda^{\prime} \lambda^{\prime \prime}} \alpha\left(\left\{\lambda^{\prime}\right\}\left\{\lambda^{\prime \prime}\right\} \rightarrow\{\lambda\}\right)\left(\{1\} \otimes\left\{\lambda^{\prime}\right\}\right)\left(\{0\} \otimes\left\{\lambda^{\prime \prime}\right\}\right. \\
& =\sum_{\lambda^{\prime} n^{\prime \prime}} \alpha\left(\left\{\lambda^{\prime}\right\}\left\{n^{\prime \prime}\right\} \rightarrow\{\lambda\}\right)\left\{\lambda^{\prime}\right\}
\end{aligned}
$$

where use was made of (33),(46) and (47). From Littlewood's rules, the Schur functions $\left\{\lambda^{\prime}\right\}$ that can couple with some symmetric Schur function $\left\{n^{\prime \prime}\right\}$ to give $\{\lambda\}$ are the ones that satisfy

$$
\lambda_{1} \geq \lambda_{1}^{\prime} \geq \ldots \geq \lambda_{n-1} \geq \lambda_{n-1}^{\prime} \geq \lambda_{n}
$$

Using this result in Eq.(72) one sees that under the restriction $U(n) \supset U(n-1)$ one has the reduction

$$
\{\lambda\}=\sum_{\lambda^{\prime}}\left\{\lambda^{\prime}\right\}
$$

in which the $\left\{\lambda^{\prime}\right\}$ are all the $U(n-1)$ irreps satisfying Eq.(73).

These are the well known in-betweeness conditions introduced by Gelfand[34] in the labeling of basis states of $U(n)$ irreps.

In the last line of (71) one has the case of a reduction of an irrep of a unitary group into irreps of its subgroup which is a direct product of one group acting in a set of variables by another that acts in the remaining ones. In this case one has the plethysm

$$
\begin{aligned}
\left(\{1\}^{\prime}\{1\}^{\prime \prime}\right) \otimes\{\lambda\} & =\sum_{\lambda^{\prime} \lambda^{\prime \prime}} \alpha\left(\left\{\lambda^{\prime}\right\} \times\left\{\lambda^{\prime \prime}\right\} \rightarrow\{\lambda\}\right)\left(\{1\}^{\prime} \otimes\left\{\lambda^{\prime}\right\}\right)\left(\{1\}^{\prime \prime} \otimes\left\{\lambda^{\prime \prime}\right\}=\right. \\
\sum_{\lambda^{\prime} \lambda^{\prime \prime}} \alpha\left(\left\{\lambda^{\prime}\right\} \times\left\{\lambda^{\prime \prime}\right\}\right. & \rightarrow\{\lambda\})\left\{\lambda^{\prime}\right\}\left\{\lambda^{\prime \prime}\right\} .
\end{aligned}
$$


The inner product $\left\{\lambda^{\prime}\right\} \times\left\{\lambda^{\prime \prime}\right\}$ in the RHS of (75) requires that the irreps $\left\{\lambda^{\prime}\right\}$ and $\left\{\lambda^{\prime \prime}\right\}$ that appear in the reduction of $\{\lambda\}$ have both the same degree as the one of $\{\lambda\}$. This result has an important role in application to Nuclear Physics in the treatment of the spin-isopin part of a system of nucleons. In this case the basis functions are labeled by the chain of subgroups

$$
U(4) \supset U(2)_{\text {spin }} \times U(2)_{i s o s p i n} .
$$

Using (75) it follows that the reduction of an irrep $\{\lambda\}$ of $U(4)$ into irreps $\{\mu\}\{\nu\}$ of $U(2) \times U(2)$ is given by

$$
\{\lambda\}=\sum_{\mu \nu}^{\oplus} \alpha(\{\mu\} \times\{\nu\} \rightarrow\{\lambda\})\{\mu\}_{\text {spin }}\{\nu\}_{\text {isospin }} .
$$

The correspondence with total spin and isospin is given by

$$
S=\frac{1}{2}\left(\mu_{1}-\mu_{2}\right) ; T=\frac{1}{2}\left(\nu_{1}-\nu_{2}\right) ; \quad \mu_{1}+\mu_{2}=\nu_{1}+\nu_{2}=n .
$$

In the treatment of systems of identical particles, in (75) one uses $\{\lambda\}=\{n\}$ for bosons and $\{\lambda\}=\left\{1^{n}\right\}$ for fermions. For these case one has

$$
\begin{aligned}
\left(\{1\}^{\prime}\{1\}^{\prime \prime}\right) \otimes\{n\} & =\sum_{\lambda}\{\lambda\}^{\prime}\{\lambda\}^{\prime \prime}, \\
\left(\{1\}^{\prime}\{1\}^{\prime \prime}\right) \otimes\left\{1^{n}\right\} & =\sum_{\lambda}\{\lambda\}^{\prime}\{\tilde{\lambda}\}^{\prime \prime},
\end{aligned}
$$

from what follows the reduction

$$
\begin{array}{rlrl}
\{n\} & \rightarrow \sum_{\lambda}\{\lambda\}^{\prime}\{\lambda\}^{\prime \prime}, & r_{\lambda^{\prime}}=r_{\lambda^{\prime \prime}}=n, \\
\left\{1^{n}\right\} \rightarrow \sum_{\lambda}\{\lambda\}^{\prime}\{\tilde{\lambda}\}^{\prime \prime}, & r_{\lambda^{\prime}}=r_{\lambda^{\prime \prime}}=n,
\end{array}
$$

where we have used the notation $\{\lambda\}^{\prime}$ and $\{\lambda\}^{\prime \prime}$ to denote partitions with no more than $n^{\prime}$ and $n^{\prime \prime}$ rows, respectively.

Consider, as another example, the reduction of the irrep $\left\{1^{n}\right\} U(4 \ell+2)$ into irreps of $S U(2) \times O^{+}(2 \ell+1)$. Using (70) and (71) one should first compute the plethysm $\left([1 / 2]^{\prime}(1)\right) \otimes\left\{1^{n}\right\}$. Using the isomorphism between $S U(2)$ and $G L(2)$ we take $[1 / 2]^{\prime}=\{1\}$. Using ( 34$)$,

$$
\alpha\left(\left\{\lambda^{\prime}\right\} \times\left\{\lambda^{\prime \prime}\right\} \rightarrow\{n\}\right)=\delta_{\left\{\lambda^{\prime \prime}\right\},\left\{\lambda^{\prime}\right\}}, \alpha\left(\left\{\lambda^{\prime}\right\} \times\left\{\lambda^{\prime \prime}\right\} \rightarrow\left\{1^{n}\right\}\right)=\delta_{\left\{\lambda^{\prime \prime}\right\},\left\{\widetilde{\lambda}^{\prime}\right\}}
$$

and (46), it follows that

$$
\begin{aligned}
\left(\{1\}^{\prime}\{1\}\right) \otimes\left\{1^{n}\right\} & =\sum_{\lambda^{\prime} \lambda^{\prime \prime}} \alpha\left(\{\lambda\}^{\prime} \times\{\lambda\}^{\prime \prime} \rightarrow\left\{1^{n}\right\}\right)\left(\{1\}^{\prime} \otimes\{\lambda\}^{\prime}\right) . \\
\cdot\left(\{1\} \otimes\{\lambda\}^{\prime \prime}\right) & =\sum_{\lambda}\{\lambda\}^{\prime}\{\tilde{\lambda}\}^{\prime \prime},
\end{aligned}
$$

where $\{\lambda\}$ are partitions of $n$. Since $\{\lambda\}$ must be expressed in terms of $G L(2)$ irreps, only the irreps $\{\lambda\}$ of type $\{p, q\}$ with $p+q=n$ contribute. Such irreps are expressed in terms of $S U(2)$ irreps as $[(p-q) / 2]$. One has then,

$$
\left\{1^{n}\right\} \rightarrow\left[\frac{n}{2}\right]\left\{1^{p}\right\}+\sum_{p>q \geq 1 ; \quad p+q=n}\left[\frac{1}{2}(p-q)\right]\left\{2^{q}, 1^{p-q}\right\}
$$

where $\left\{1^{p}\right\}$ and $\left\{2^{q}, 1^{p-q}\right\}$ must be expressed in terms of $O^{+}(7)$ irreps. As an example, for $n=3$, one has

$$
\left\{1^{3}\right\} \rightarrow\left[\frac{3}{2}\right]^{\prime}\{111\}+\left[\frac{1}{2}\right]^{\prime}\{210\}=\left[\frac{3}{2}\right]^{\prime}[111]+\left[\frac{1}{2}\right]^{\prime}([21]+[1])
$$


where use is made of (84) [given below] to express the $U(7)$ irreps in terms of the ones of $O^{+}(7)$.

In cases in which $f 1\}=+1+$, due to property (46), the theorem gives a trivial identity being of no use. It is what happens, for example, in the reduction $U(n) \rightarrow O(n)$. For this case one has the known result $[1,2,3]$ :

" The character $\{\lambda\}$ of $U(n)$ decomposes into $O(n)$ characters $\left(\lambda^{\prime \prime}\right)$ by the relation

$$
\{\lambda\}=\sum_{\lambda^{\prime \prime}}\left[\sum_{\lambda^{\prime}} \alpha\left(\left\{\lambda^{\prime}\right\}\left\{\lambda^{\prime \prime}\right\} \rightarrow\{\lambda\}\right)\right]\left(\lambda^{\prime \prime}\right)
$$

where the sum is made in the irreps $\left\{\lambda^{\prime}\right\}$ with even components." For example,

$$
\begin{aligned}
\{0\} & =(0) ; \quad\{1\}=(1) ; \quad\{2\}=(2)+(0) ; \\
\{311\} & =(311)+(21)+(111) .
\end{aligned}
$$

Using a table of reduction $U(n) \rightarrow O(n)$ [ One such a table can be found, for instance, in Ref.[3]. ] one can obtain the inverse result: to express the characters of an $O(n)$ irrep $(\lambda)$ in terms of $U(n)$ irreps $\{\nu\}$. A general procedure to express the characters of $O(n)$ in terms of those of $U(n)$ is given in Ref.[9]
The plethysm technique can be applied in the various versions of the Interacting Boson Model(IBM) which have a very rich algebraic structure. For example, in IBM-4 one needs the branching rule for the reduction of irreps $\{\lambda\}$ of $U_{S T}(6)$ into irreps $\{\mu\}\{\nu\}$ of the semi-direct product $U_{S}(t) \oslash U_{T}(3)$ of $U_{S}(t)$ by $U_{T}(3)$. This reduction is obtained using the 9 th line of Eq.(71). Proceeding symilarly to the case $U(\mathrm{~nm}) \supset U(n) \times U(m)$ one obtains for this reduction the result

$$
\{\lambda\}_{S T}=\sum_{\{\mu\}_{S}\{\nu\}_{T}} \alpha\left(\{\mu\}_{S}\{\nu\}_{T} \rightarrow\{\lambda\}_{S T}\right)\{\mu\}_{S}\{\nu\}_{T}
$$

$\{\mu\}_{S}$ and $\{\nu\}_{T}$ being $U(3)$ irreps.

\section{IV.1 Aplication in Nuclear Structure}

In the traditional nonrelativistic treatment the nucleus is considered as a system of $A$ fermions, the nucleons, with spin and isospin $1 / 2$ and 3 spatial degrees of freedom interacting through one- and two-body forces. The bound states of such system are described by totally antisymmetric wave functions since its constituents are fermions.

The introduction of Jacobi vectors

$$
\begin{aligned}
\vec{\rho}_{i} & =\frac{1}{\sqrt{i(i+1)}}\left(\sum_{j=1}^{i} \vec{r}_{j}-i \vec{r}_{i+1}\right) ; i=1,2, \ldots, A-1, \\
\vec{\rho}_{A} & =\frac{1}{\sqrt{A}} \sum_{j=1}^{A} \vec{r}_{j}
\end{aligned}
$$

allows us to remove the center of mass and focus attention only to the relative motion described by the translationally invariant Jacoby vectors $\vec{\rho}_{1}, \vec{\rho}_{2}, \ldots, \vec{\rho}_{A-1}$.

To describe the bound states of such system we will use as basis the basis functions of irrep $\left[1^{7(A-1)}\right]$ of $U(7(A-$ 1)) $\supset U^{(r)}(3(A-1)) \times U^{(s)}(4(A-1))$. The spin-isospin part is described using the chain

$$
\begin{array}{llll}
U^{(s)}(4(A-1)) & U^{(S T)}(4) & \times & U^{(s)}(A-1) \\
& \cup & \cup \\
& U^{(S)}(2) \times U^{(T)}(2) & & O^{(s)}(A-1) \\
& & \cup \\
& & \mathrm{S}^{(s)}(A)
\end{array}
$$

while the space part is described by

$$
\begin{array}{cllll}
\mathrm{U}^{(r)}(3(A-1) & \supset & \mathrm{U}(3) & \times & \mathrm{U}^{(r)}(A-1) \\
& \cup & & \cup \\
& \mathrm{O}^{+}(3) & & \mathrm{O}^{(r)}(A-1) \\
& & & \cup \\
& & \mathrm{S}^{(r)}(A) .
\end{array}
$$


It follows then the interest in studying the branching rules of irreps of chains of kind

$$
U(A-1) \supset O(A-1) \supset S(A) .
$$

This notation is a bit misleading since it may raise the question:how is it possible that the permutation group of $A$ objects may be a subgroup of a transformation group of a smaller number $A-1$ of objects? The answer is that the objects are not the same. In $U(A-1)$ and $O(A-1)$ we consider the radial components of the $A-1$ translationally invariant Jacobi vectors $(86)$ while in $S^{(r)}(A)$ the objects are the radial components of the position vectors $\vec{r}_{i}$. A permutation of the position vectors $\vec{r}_{i}$ turns out to be a orthogonal transformation of the translationally invariant Jacobi vector and therefore one has $O^{(r)}(A-1) \supset S^{(r)}(A)$.

Since the basis functions of the irrep $\{\lambda\}$ of $U^{(r)}(3(A-1))$ are functions only of the coordinates of the first $A-1$ Jacobi vectors, $\{\lambda\}$ has to be symmetric. We then write $\{\lambda\}=\{E\}$. Since the wave functions of the $p$-dimensional harmonic oscillator carry the irrep $\{E\}$ of $U(p)$, it is usual to associate $E$ to the configuration energy of the nuclear states whose space part is described by wave functions labeled by the chain (89).

This association allows us to stablish a link with the harmonic oscillator shell model. The basis functions of the irrep $\{E\}$ could alternatively be labeled by the chain of subroups

$$
U(3(A-1)) \supset U^{(1)}(3) \times U^{(2)}(3) \times \ldots \times U^{(A-1)}(3)
$$

in which each link $U^{(i)}(3)$ acts only in the 3 coordinates of the Jacoby vector $\vec{\rho}_{i}$. In this case the irreps associated to those $U^{(i)}(3)$ would be all symmetric $\left[E^{(i)}\right]$ and their basis functions would be eigenstates of harmonic oscillators with energy $\mathcal{E}^{(i)}=\left(E^{(i)}+3 / 2\right) \hbar \omega$ and it would result

$$
E=\sum_{i=1}^{A-1} E^{(i)}
$$

The number of linearly independent wave functions of the 3-dimensional harmonic oscillator with energy
$\mathcal{E}=(E+3 / 2) \hbar \omega$ is equal to the dimension of the irrep $\{E\}$ of $U(3)$ given by

$$
\operatorname{dim}_{\{E\}}=\frac{1}{2}(E+1)(E+2) .
$$

In this way, by Pauli principle, in the $E$ shell one can put at most $4 \operatorname{dim}_{\{E\}}=2(E+1)(E+2)$ nucleons. The minimal configuration energy is obtained by filling the shells $E=0(s), E=1(p), E=2(s-d), \ldots, E_{0}-1$ and putting the remaining $n_{0}$ nucleons in the first partially filled shell $E_{0}$. The fillled shells(core) will contain

$$
n_{\text {core }}\left(E_{0}\right)=\sum_{E=0}^{E_{0}-1} 2(E+1)(E+2)=\frac{2}{3} E_{0}\left(E_{0}+1\right)\left(E_{0}+2\right)
$$

nucleons. This equation allows one to find, for a given $A$, the value of $E_{0}$ as the one for which $n_{\text {core }}\left(E_{0}\right) \leq A \leq$ $n_{\text {core }}\left(E_{0}+1\right)$. Then we will have

$$
E_{\min }=\sum_{E=0}^{E_{0}-1} 4 E \operatorname{dim}_{\{E\}}+E_{0}\left(A-A_{\text {core }}\left(E_{0}\right)\right)=E_{0} A-\frac{1}{6} E_{0}\left(E_{0}+1\right)\left(E_{0}+2\right)\left(E_{0}+3\right) .
$$

Our aim is to label the states of a system of $A$ nucleons with minimal configuration energy $E=E_{\text {min }}$ with the labels given by the unitary chain (89). This labelling has been extensively used in our applications of the Restricted Dynamics Model[39].
The $U(3(A-1))$ irreps are labeled by $E$. For $1 \leq A \leq 4$ one has $E_{0}=0$ and $E=0$. For $A \geq 5$ we put $E=E_{\min }$ obtained by use of Eqs.(94) and (95).

The $U(3)$ irreps are labeled by 3 integers 
$\left\{E_{1}, E_{2}, E_{3}\right\}$ that, according to (78) must satisfy

$$
E_{1}+E_{2}+E_{3}=E .
$$

Also, for (78), the $U^{(r)}(A-1)$ irreps are the same of $U(3)$, that is, $\left\{E_{1}, E_{2}, E_{3}\right\}$. Eq.(84) give us the $O^{(r)}(A-1)$ irreps contained in the $U^{(r)}(A-1)$ irrep $\left\{E_{1}, E_{2}, E_{3}\right\}$. One of them is by sure $\left(E_{1}, E_{2}, E_{3}\right)$ and only this corresponds to states of minimal configuration energy. This fix us the $O^{(r)}(A-1)$ irrep as that of $U^{(r)}(A-1)$. It still remains the restriction $O^{(r)}(A-1) \rightarrow S^{(r)}(A)$.

The $U^{(r)}(A-1)$ irreps $\left\{E_{1}, E_{2}, E_{3}\right\}$ are all the ones that satisfy (96) . The Pauli principle, however, imposes an additional restriction. The treatment of the spin-isospin part by the Wigner supermultiplet model implies that the $S^{(s)}(A)$ irrep $[\widetilde{\lambda}]$ must have at most 4 lines, that is,

$$
[\tilde{\lambda}]=\left[\tilde{\lambda}_{1}, \tilde{\lambda}_{2}, \tilde{\lambda}_{3}, \tilde{\lambda}_{4}\right] .
$$

Therefore the $S^{(r)}(A)$ irrep $[\lambda]$, being its conjugate, must have at most 4 columns.

We will see in the following the branching rules in the chain $U(A-1) \supset S(A)$. The reduction of irreps in the chain $O(A-1) \supset S(A)$ is obtained by first expressing the $O(A-1)$ characters in terms of those of $U(A-1)$ and , after, making the reduction $U(A-1) \rightarrow S(A)$ of the resulting $U(A-1)$ irreps.

The $S(A)$ irreps contained in the reduction of the $U(A-1)$ irrep $\left\{\lambda^{\prime}\right\}$ are given by the expansion of the inner plethysm $\{A-1,1\} \odot\left\{\lambda^{\prime}\right\}$ in terms of $S(A)$ irreps [Refs.[13, 38]]:

$$
\{A-1,1\} \odot\left\{\lambda^{\prime}\right\}=\sum_{\lambda^{\prime \prime}} V_{\lambda^{\prime} \lambda^{\prime \prime}}\left[A-r^{\prime \prime}, \lambda_{1}^{\prime \prime}, \lambda_{2}^{\prime \prime}, \ldots, \lambda_{A-1}^{\prime \prime}\right]
$$

where $r^{\prime \prime}=\sum_{i} \lambda_{i}^{\prime \prime}$ is the degree of $U(A-1)$ irrep $\left\{\lambda^{\prime \prime}\right\}$.

To obtain the multiplicity coefficients $V_{\lambda^{\prime} \lambda^{\prime \prime}}$ that appear in (98) one needs first to define the operators $\widehat{D}$ and $\widehat{\mathcal{D}}$ which act on $U(A-1)$ irreps

The operator $\widehat{\mathcal{D}}(\{\lambda\})$ is defined by its action over an irrep $\left\{\lambda^{\prime}\right\}$ :

$$
\widehat{\mathcal{D}}(\{\lambda\})\left\{\lambda^{\prime}\right\}=\sum_{\lambda^{\prime \prime}} \alpha\left(\{\lambda\}\left\{\lambda^{\prime \prime}\right\} \rightarrow\left\{\lambda^{\prime}\right\}\right)\left\{\lambda^{\prime \prime}\right\} .
$$

As example, $\widehat{\mathcal{D}}(\{2\})\{31\}=\{2\}+\left\{1^{2}\right\}$.

From the properties of the outer product of Schur functions, it follows that the operators $\widehat{\mathcal{D}}$ satisfy the relations:

$$
\begin{aligned}
\widehat{\mathcal{D}}\left(\left\{\lambda^{\prime}\right\}\right) \widehat{\mathcal{D}}\left(\left\{\lambda^{\prime \prime}\right\}\right) & =\widehat{\mathcal{D}}\left(\left\{\lambda^{\prime}\right\}\left\{\lambda^{\prime \prime}\right\}\right), \\
\widehat{\mathcal{D}}\left(\left\{\lambda^{\prime}\right\}\right)+\widehat{\mathcal{D}}\left(\left\{\lambda^{\prime \prime}\right\}\right) & =\widehat{\mathcal{D}}\left(\left\{\lambda^{\prime}\right\}+\left\{\lambda^{\prime \prime}\right\}\right) .
\end{aligned}
$$

The operator $\widehat{D}$ is defined in term of $\widehat{\mathcal{D}}$ by

$$
\begin{aligned}
\widehat{D}= & \sum_{t_{2}=0}^{\infty} \sum_{\lambda_{t_{2}}} \sum_{t_{3}=0}^{\infty} \sum_{\lambda_{t_{3}}} \ldots \sum_{j_{2}=0}^{\infty} \sum_{j_{3}=0}^{\infty} \ldots\left(\{\lambda\}_{t_{2}}\{\lambda\}_{t_{3}} \ldots\right)\left(\widehat{\mathcal{D}}\left(\{2\} \otimes\{\lambda\}_{t_{2}}\right) .\right. \\
& \cdot\left(\widehat { \mathcal { D } } ( \{ 3 \} \otimes \{ \lambda _ { t _ { 3 } } \} ) \ldots \left(\widehat { \mathcal { D } } ( \{ 2 \} \otimes \{ j _ { 2 } \} ) \left(\widehat{\mathcal{D}}\left(\{3\} \otimes\left\{j_{3}\right\}\right) \ldots .\right.\right.\right.
\end{aligned}
$$

The $\left\{j_{2}\right\},\left\{j_{3}\right\}, \ldots$ are symmetric Schur functions and the $\{\lambda\}_{t_{2}},\{\lambda\}_{t_{3}}, \ldots$ are general Schur functions of degrees $t_{2}, t_{3}, \ldots$.

Denoting by $r, r^{\prime}$ and $r^{\prime \prime}$ the degrees of $\{\lambda\},\left\{\lambda^{\prime}\right\}$ and $\left\{\lambda^{\prime \prime}\right\}$ one sees from (99) that the Schur functions $\left\{\lambda^{\prime \prime}\right\}$ produced by $\widehat{\mathcal{D}}(\{\lambda\})$ when acting in $\left\{\lambda^{\prime}\right\}$ have degrees $r^{\prime \prime}=r^{\prime}-r$. Since $r^{\prime \prime}$ must be nonnegative, this limits the sums in $t_{2}, t_{3}, \ldots$ when $\widehat{D}$ acts in an irrep $\left\{\lambda^{\prime}\right\}$.

By property (101) it follows that

$$
\begin{aligned}
\widehat{\mathcal{D}}\left(\{k\} \otimes\{\lambda\}_{t_{k}}\right) & =\widehat{\mathcal{D}}\left(\sum_{\lambda^{\prime \prime}} \alpha\left(\{k\} \otimes\{\lambda\}_{t_{k}} \rightarrow\left\{\lambda^{\prime \prime}\right\}\right)\left\{\lambda^{\prime \prime}\right\}\right)= \\
\sum_{\lambda^{\prime \prime}} \alpha\left(\{k\} \otimes\{\lambda\}_{t_{k}}\right. & \left.\rightarrow\left\{\lambda^{\prime \prime}\right\}\right) \widehat{\mathcal{D}}\left(\left\{\lambda^{\prime \prime}\right\}\right)
\end{aligned}
$$


for each plethysm that appears in (102). After, by use of (100), the product of $\widehat{\mathcal{D}}$ 's is converted into a single $\widehat{\mathcal{D}}$ with argument equal to the product of the Schur functions of each factor. Making the outer product of these Schur functions and using again (101) one can rewrite $\widehat{D}$ in a form linearized in the $\widehat{\mathcal{D}}$ 's and in the Schur functions:

$$
\widehat{D}=\sum_{\lambda^{\prime} \lambda^{\prime \prime}} C_{\lambda^{\prime} \lambda^{\prime \prime}}\left\{\lambda^{\prime}\right\} \widehat{\mathcal{D}}\left(\left\{\lambda^{\prime \prime}\right\}\right)
$$

the $C_{\lambda^{\prime}} \lambda^{\prime \prime}$ being only numerical coefficients.

In the linearized form of $\widehat{D}$ one can gather the terms whose $\widehat{\mathcal{D}}$ 's arguments have the same degree, that is,

$$
\widehat{D}=\sum_{i=0}^{\infty}\left[\sum_{\{\lambda\}_{i}}\left(\sum_{\lambda^{\prime \prime}} C_{\lambda^{\prime \prime}\{\lambda\}_{i}}\left\{\lambda^{\prime \prime}\right\}\right) \widehat{\mathcal{D}}\left(\{\lambda\}_{i}\right)\right] \equiv \sum_{i=0}^{\infty} \delta_{i}
$$

When $\widehat{D}$ acts over $\left\{\lambda^{\prime}\right\}$ one obtains

$$
\widehat{D}\left\{\lambda^{\prime}\right\}=\sum_{i=0}^{\infty} \delta_{i}\left\{\lambda^{\prime}\right\}=\sum_{i=0}^{\infty}\left[\sum_{\{\lambda\}_{i}}\left(\sum_{\lambda^{\prime \prime}} C_{\lambda^{\prime \prime}\{\lambda\}_{i}}\left\{\lambda^{\prime \prime}\right\}\right) \widehat{\mathcal{D}}\left(\{\lambda\}_{i}\right)\left\{\lambda^{\prime}\right\}\right] .
$$

Using (99), performing the outer products, and collecting similar terms one obtains a linear expression in the Schur functions

$$
\widehat{D}\left\{\lambda^{\prime}\right\}=\sum_{\lambda^{\prime \prime}} V_{\lambda^{\prime}} \lambda^{\prime \prime}\left\{\lambda^{\prime \prime}\right\} .
$$

This final reduction gives us the irreps $\left\{\lambda^{\prime \prime}\right\}$ and the numerical coefficients $V_{\lambda^{\prime}} \lambda^{\prime \prime}$ which appear in (98). The $\left\{\lambda^{\prime \prime}\right\}$ 's give the $S(A)$ irreps $\left[A-r^{\prime \prime}, \lambda_{1}^{\prime \prime}, \lambda_{2}^{\prime \prime}, \ldots, \lambda_{A}^{\prime \prime}\right]$ present in the reduction of $U(A-1)$ irrep $\left\{\lambda^{\prime}\right\}$ and the $V$ 's are their multiplicities.
From the procedure described above, to obtain the $\delta_{1}$ 's given in (104) the only $t_{k}$ and $j_{k}$ in (102) that contribute for $\delta_{i}$ are the solutions by nonnegative integers of the equation

$$
\sum_{k=2}^{\infty} k\left(t_{k}+j_{k}\right)=i
$$

For $i=0$ only one solution exists, $t_{k}=j_{k}=0$, and therefore, $\delta_{1}=0$. For $i=1$ there is no solution, then $\delta_{1}=0$. For $i=2,3$ and 4 the following results follow:

$$
\begin{aligned}
\delta_{2}= & (\{0\}+\{1\}) \widehat{\mathcal{D}}(\{2\}), \quad \delta_{3}=(\{0\}+\{1\}) \widehat{\mathcal{D}}(\{3\}), \\
\delta_{4}= & (2\{0\}+2\{1\}+\{2\}) \widehat{\mathcal{D}}(\{4\})+\left(\{1\}+\left\{1^{2}\right\}\right) \widehat{\mathcal{D}}(\{31\})+ \\
& +(\{0\}+\{1\}+\{2\}) \widehat{\mathcal{D}}\left(\left\{2^{2}\right\}\right) .
\end{aligned}
$$

From the definition of the $\widehat{\mathcal{D}}$ 's it follows that $\delta_{i}\left\{\lambda^{\prime}\right\}=0$ when the degree of $\left\{\lambda^{\prime}\right\}$ is less than $i$ and, therefore, it results that

$$
\widehat{D}\left\{\lambda^{\prime}\right\}_{r}=\left(\delta_{0}+\delta_{1}+\ldots+\delta_{r}\right)\left\{\lambda^{\prime}\right\}_{r}
$$

for a Schur function of degree $r$. With the $\delta$ 's listed above one can compute the reduction $U(n-1) \rightarrow S(n)$ for irreps of degree less or equal to 4 :

$$
\begin{aligned}
\{0\} & =[n], \\
\{1\} & =[n-1,1], \\
\{2\} & =[n-2,2]+[n-1,1]+[n], \\
\left\{1^{2}\right\} & =\left[n-2,1^{2}\right], \\
\{3\} & =[n-3,3]+[n-2,2]+\left[n-2,1^{2}\right]+2[n-1,1]+[n], \\
\{21\} & =[n-3,2,1]+[n-2,2]+\left[n-2,1^{2}\right]+[n-1,1],
\end{aligned}
$$




$$
\begin{aligned}
\left\{1^{3}\right\}= & {\left[n-3,1^{3}\right], } \\
\{4\}= & 2[n]+3[n-1,1]+3[n-2,2]+\left[n-2,1^{2}\right]+[n-3,3] \\
& +[n-3,2,1]+[n-4,4], \\
\{31\}= & 2[n-1,1]+2[n-2,2]+3\left[n-2,1^{2}\right]+[n-3,3]+[n-3,2,1] \\
& +\left[n-3,1^{3}\right]+[n-4,3,1], \\
\left\{2^{2}\right\}= & {[n]+[n-1,1]+2[n-2,2]+[n-3,3]+[n-3,2,1,]+\left[n-4,2^{2}\right], } \\
\left\{21^{2}\right\}= & {\left[n-2,1^{2}\right]+[n-3,2,1]+\left[n-3,1^{3}\right]+\left[n-4,2,1^{2}\right], } \\
\left\{1^{4}\right\}= & {\left[n-4,1^{4}\right] . }
\end{aligned}
$$

Using a table of reduction $U(n) \supset O(n)$ [Ref.[3]], one can express, by subtractions, the characters of $O(n)$ in terms of those of $U(n)$.

In Refs. [3, 38] there is a table of the reduction $O(n-1) \supset S(n)$ for $\left(\lambda^{\prime}\right)$ of degree no more than 7 and with no more than 3 rows.

The expansion (104) of $\widehat{D}$ in terms of $\delta_{i}$ allows us to compute all the $S(A)$ irreps $\left[\lambda^{\prime \prime}\right]$ contained in the $U(A-1)$ irrep $\left\{\lambda^{\prime}\right\}$. For great values of $r_{\lambda^{\prime}}$ this process is too much laborious.

In applications in Nuclear Physics one has that the $U(A-1)$ irreps of interest are, according to (96), the ones with no more than 3 rows, that is, those of the form $\left\{\lambda^{\prime}\right\}=\left\{E_{1}, E_{2}, E_{3}\right\}$. Besides, Pauli principle imposes that the only physically acceptable $S(A)$ irreps are those of the form

$$
[\lambda]=\left[4^{k_{4}}, 3^{k_{3}}, 2^{k_{2}}, 1^{k_{1}}\right]
$$

with

$$
4 k_{4}+3 k_{3}+2 k_{2}+k_{1}=A .
$$

The $k_{i}$ are interpreted[36] as the number of space levels occupyed by $1,2,3$, and 4 nucleons, respectively.

From now on we will restrict ourselves to the cases of interest in Nuclear Physics, that is, the ones in which the $U(A-1)$ irreps $\left\{\lambda^{\prime}\right\}$ are also $U(3)$ irreps satisfying Eq.(96) and the $S(A)$ irreps $[\lambda]$ satisfy (110) This means that $\left\{\lambda^{\prime}\right\}$ has at most 3 rows and $[\lambda]$ at most 4 columns. These restrictions, as will be seen below, simplify enormously the calculations.

Having in mind these restrictions, let us find which $t_{k}$ 's and $j_{k}$ 's in Eq.(102) may give a contribution. When applying the linearized form [Eq.(103)] of $\widehat{D}$ to a Schur function $\{\lambda\}_{E}^{(A)}$ of $U(A-1)$ one has, using Eqs. (99) and (103),

$$
\widehat{D}\{\lambda\}_{E}^{(A)}=\sum_{\lambda^{\prime} \lambda^{\prime \prime}} C_{\lambda^{\prime}} \lambda^{\prime \prime} \sum_{\nu} \alpha\left(\left\{\lambda^{\prime \prime}\right\}\{\nu\} \rightarrow\{\lambda\}_{E}^{(A)}\right) \sum_{\lambda^{\prime \prime \prime}} \alpha\left(\left\{\lambda^{\prime}\right\}\{\nu\} \rightarrow\left\{\lambda^{\prime \prime \prime}\right\}\right)\left\{\lambda^{\prime \prime \prime}\right\}
$$

By Eq.(98) the Schur functions $\left\{\lambda^{\prime \prime \prime}\right\}$ will give $S(A)$ irreps

$$
[\lambda]=\left[A-r_{\lambda^{\prime \prime \prime}}, \lambda_{1}^{\prime \prime \prime}, \lambda_{2}^{\prime \prime \prime}, \ldots, \lambda_{A-1}^{\prime \prime \prime}\right] .
$$

[Here we recall that $r_{\mu}$ denotes the degree of a general Schur function $\{\mu\}$.]

Since $\{\lambda\}_{E}^{(A)}$ has at most 3 rows, the outer product $\left\{\lambda^{\prime \prime}\right\}\{\nu\}$ gives a nonzero contribution only when both $\left\{\lambda^{\prime \prime}\right\}$ and $\{\nu\}$ have at most 3 rows, i.e.

$$
\left\{\lambda^{\prime \prime}\right\}=\left\{\lambda_{1}^{\prime \prime}, \lambda_{2}^{\prime \prime}, \lambda_{3}^{\prime \prime}\right\} \text { and }\{\nu\}=\left\{\nu_{1}, \nu_{2}, \nu_{3}\right\}
$$

Since $\left\{\lambda^{\prime \prime}\right\}$ is a component of the reduction

$$
\left(\{2\} \otimes\{\lambda\}_{t_{2}}\right)\left(\{2\} \otimes\left\{j_{2}\right\}\right)\left(\{3\} \otimes\{\lambda\}_{t_{3}}\right)\left(\{3\} \otimes\left\{j_{3}\right\}\right) \ldots,
$$

only contribute the components in $\{k\} \otimes\{\lambda\}_{t_{k}}$ and $\{k\} \otimes\left\{j_{k}\right\}$ with no more than 3 rows.

We then introduce the notation $\{m\} \dot{\otimes}\{\mu\}$ to denote what we call a reduced plethysm:

$$
\{m\} \dot{\otimes}\{\mu\}=\text { only terms of }\{m\} \otimes\{\mu\} \text { with no more than } 3 \text { rows. }
$$


In our applications to Nuclear Physics the only plethysms that one needs to compute are the reduced ones. These have much less components than the complete plethysms, what makes the computer calculations feasible.

The rules of outer product are such that if one of its factors has more than $p$ rows, the same occurs with all the Schur functions in its reduction. Due to that, all the plethysms properties here presented, but Eq.(39), apply to reduced plethysms.

Computer calculations using Eq.(60) show that

$$
\{k\} \dot{\otimes}\left\{1^{m}\right\}=0, \text { for } m>(k+1)(k+2) / 2 .
$$

From this result one proves that

$$
\begin{aligned}
\{k\} \dot{\otimes}\{\mu\}= & 0 \text { for Schur functions }\{\mu\} \text { with more than } \\
& m=(k+1)(k+2) / 2 \text { rows. }
\end{aligned}
$$

In order to produce a physically acceptable contribution, the Schur functions $\left\{\lambda^{\prime}\right\}$ in Eq.(112) when outer multiplyed by $\{\nu\}$ must give, by Eq.(98) and (110), in its reduction a Schur function with at most 4 columns. By the rules of outer product, it follows that both $\left\{\lambda^{\prime}\right\}$ and $\{\nu\}$ must have this property:

$$
\left\{\tilde{\lambda}^{\prime}\right\}=\left\{\bar{\lambda}_{1}^{\prime}, \bar{\lambda}_{2}^{\prime}, \bar{\lambda}_{3}^{\prime}, \bar{\lambda}_{4}^{\prime}\right\} ;\{\tilde{\nu}\}=\left\{\bar{\nu}_{1}, \bar{\nu}_{2}, \bar{\nu}_{3}, \bar{\nu}_{4}\right\} \text {. }
$$

Since $\left\{\lambda^{\prime}\right\}$ is a term of the reduction of $\left\{\lambda_{t_{2}}\right\}\left\{\lambda_{t_{3}}\right\} \ldots$, it follows that also the $\{\lambda\}_{t_{k}}$ must have this property.

This implies that among the reduced plethysms $\{k\} \dot{\otimes}\{\lambda\}_{t_{k}}$ that appear in $\mathrm{Eq}(102)$, only those in which the length of the $\{\lambda\}_{t_{k}}$ 's rows do not exceed 4 must be considered.

The analysis of degrees of the Schur functions in Eq.(112) gives further informations:

$$
r_{\lambda^{\prime \prime}}+r_{\nu}=E ; r_{\lambda^{\prime}}+r_{\nu}=r_{\lambda^{\prime \prime \prime}} .
$$

From Eq.(113) and (110) one has

$$
A-r_{\lambda^{\prime \prime \prime}} \leq 4 \text {. }
$$

Combining Eq.(119) and (120) it follows that

$$
i \equiv r_{\lambda^{\prime \prime}}-r_{\lambda^{\prime}} \leq E-A+4
$$

what sets an upper bound in $i$ :

$$
i_{\max }=E-A+4 .
$$

From Eq.(114) and (118) one concludes that

$$
r_{\nu} \leq 12
$$

This result together with the first of Eqs.(119) implies that

$$
r_{\lambda^{\prime \prime}} \geq E-12 \text {. }
$$

Combining Eq.(102) and (103) one has

$$
r_{\lambda^{\prime}}=\sum_{k=2} t_{k} ; r_{\lambda^{\prime \prime}}=\sum_{k=2} k\left(t_{k}+j_{k}\right)
$$

and therefore

$$
i=\sum_{k=2}\left[k\left(t_{k}+j_{k}\right)-t_{k}\right]=t_{2}+\sum_{k=2} k\left(t_{k+1}+j_{k}\right) .
$$

From these equations one concludes that

$$
i \geq \sum_{k=2} t_{k}
$$

and

$i=\sum_{k=2} t_{k} \Rightarrow\left\{\begin{array}{l}t_{2}=i ; \quad t_{3}=t_{4}=\ldots=0 \\ j_{2}=j_{3}=\ldots=0\end{array}\right.$

Eq.(127), combined with Eq.(125) and (124) gives

$$
E-12 \leq r_{\lambda^{\prime \prime}} \leq 2 i
$$

from which one concludes that

$$
2 i \geq E-12 \text {. }
$$

This gives a lower bound to $i$ :

$$
i_{\text {min }}=\left[\frac{E+1}{2}\right]-6 .
$$

Summarizing: given $E$ and $A$, the $t_{k}$ 's and $j_{k}$ 's in Eq.(102) applied to $\{\lambda\}_{E}^{(A)}$ that may give physically acceptable $S(A)$ irreps are obtained following the 2 steps:

1 ) take $i$ in the range

$$
\left[\frac{E+1}{2}\right]-6 \leq i \leq E-A+4
$$


2 ) for each $i$ in this range, find the nonnegative integers $j_{k}$ and $t_{k}$ that satisfy

$$
\begin{aligned}
& t_{2}+\sum_{k=2} k\left(t_{k+1}+j_{k}\right)=i, \\
& \sum_{k=2} k\left(t_{k}+j_{k}\right)=r_{\lambda^{\prime \prime}},
\end{aligned}
$$

where $r_{\lambda^{\prime \prime}}$ must be in the range given by Eq.(129).

These conditions reduces drastically the number of $j_{k}$ 's and $j_{k}$ 's that may contribute and also their values, being valid for any value of $A$ and $E \geq E_{\text {min }}$.

Once the $t_{k}$ 's and $j_{k}$ 's that have a chance to contribute are determined, one computes the reduced plethysms associated to them and continues the calculations.

In any physical application one starts by examining first the case $E=E_{\text {min }}$, so it deserves a special attention.

For $p$-nuclei $(5 \leq A \leq 16)$ one has $E_{0}=1$ and, according to Eq. ( 95$), \bar{E}_{\min }=A-4$. In this case Eq.(132) gives only the value 0 for $i$, and by Eq.(133) one has

$$
t_{2}=t_{3}=\ldots=0 ; j_{2}=j_{3}=\ldots=0 \quad(p-\text { nuclei }) .
$$

Therefore

$$
\widehat{D}\{\lambda\}_{A-4}^{(A)} \stackrel{\circ}{=}\{\lambda\}_{A-4}^{(A)}
$$

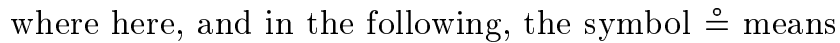
that one considers in the RHS only those Schur functions that produce physically acceptable $S(A)$ irreps.

Then, by $\operatorname{Eq}(98)$, for $p$-nuclei in ground configuration, one has the $U(A-1) \supset S(A)$ reduction

$$
\{\lambda\}_{A-4}^{(A)} \stackrel{\circ}{=}\left[4, \lambda_{1}, \lambda_{2}, \lambda_{3}\right] \quad p-\text { nuclei }
$$

with

$$
\lambda_{1}+\lambda_{2}+\lambda_{3}=A-4 \quad \text { and } \quad \lambda_{i} \leq 4 .
$$

In Appendix $\mathrm{A}$ one lists the reduction $U(A-1) \supset$ $S(A)$ for $p$-nuclei in ground configuration. One observes that the results present the symmetry under particle-hole exchange in the open shell:

$$
\begin{aligned}
A & \leftrightarrow \bar{A}=n_{\text {core }}+n_{0}-A \\
(p & \left.\equiv E_{1}-E_{2} ; \quad q \equiv E_{2}-E_{3}\right) \leftrightarrow(\bar{p}, \bar{q})=(q, p) .
\end{aligned}
$$

For this shell these symmetry relations read as

$$
\begin{aligned}
A & \leftrightarrow \bar{A}=20-A \\
{[\lambda] } & \equiv\left[4, \lambda_{1}^{(0)}, \lambda_{2}^{(0)}, \lambda_{3}^{(0)}\right] \rightarrow[\bar{\lambda}]=\left[4,4-\lambda_{3}^{(0)}, 4-\lambda_{2}^{(0)}, 4-\lambda_{1}^{(0)}\right] .
\end{aligned}
$$

For $(s-d)$-nuclei $(17 \leq A \leq 40), \quad E_{0}=2$ and $E_{\min }=2 A-20$. Eq.(132) gives again only one solution for $i$ :

$$
i=i_{\min }=i_{\max }=A-16 . \quad(s-d) \text {-nuclei }
$$

Since $i=i_{\max }$, by Eq.(128) one has the result

$$
t_{2}=A-16 ; \quad t_{3}=t_{4}=\ldots=0 ; \quad j_{2}=j_{3}=\ldots=0(s-d)-\text { nuclei }
$$

and therefore

$$
\begin{gathered}
\widehat{D}\{\lambda\}_{2 A-20}^{(A)} \stackrel{\circ}{=} \sum_{\{\lambda\}_{A-16}}\{\lambda\}_{A-16} \widehat{\mathcal{D}}\left(\{2\} \dot{\otimes}\{\lambda\}_{A-16}\right)\{\lambda\}_{2 A-20}^{(A)}=\sum_{\{\lambda\}_{A-16}}\{\lambda\}_{A-16} \\
\sum_{\{\lambda\}_{2(A-16)}} \alpha\left(\{2\} \dot{\otimes}\{\lambda\}_{A-16} \rightarrow\{\lambda\}_{2(A-16)}\right) \widehat{\mathcal{D}}\left(\{\lambda\}_{2(A-16)}\right)\{\lambda\}_{2 A-20}^{(A)},
\end{gathered}
$$

for $(s-d)$-nuclei.

The only reduced plethysms that one needs to compute, for the whole shell, are $\{2\} \dot{\otimes}\{\mu\}_{m}$ with $m=1,2, \ldots, 24$.

As seen previously,only contribute the Schur functions $\{\lambda\}_{A-16}$ with no more than $(2+1)(2+2) / 2=6$ rows and no more than 4 columns.

When acting on $\{\lambda\}_{2 A-20}^{(A)}$, the operator $\widehat{\mathcal{D}}\left(\{\lambda\}_{2(A-16)}\right)$ gives, by Eqs.(114),(123)

$$
\widehat{\mathcal{D}}\left(\{\lambda\}_{2(A-16)}\right)\{\lambda\}_{2 A-20}^{(A)} \stackrel{\circ}{=}\left(\{\lambda\}_{2(A-16)}\left\{4^{3}\right\} \rightarrow\{\lambda\}_{2 A-20}^{(A)}\right)\left\{4^{3}\right\} .
$$


The outer product of $\{\lambda\}_{2(A-16)}$ by $\left\{4^{3}\right\}$ gives only one Schur function with 3 rows $\left\{4^{3}\right\}+\{\lambda\}_{2(A-16)}$ (with multiplicity 1), and must be equal to $\{\lambda\}_{2(A-20)}$. This fixes $\{\lambda\}_{2(A-16)}$ as $\{\lambda\}_{2(A-16)}=\{\lambda\}_{2 A-20}^{(A)}-\left\{4^{3}\right\}$.

On the other hand, the outer product $\{\lambda\}_{A-16}\left\{4^{3}\right\}$ has only one component with up to 4 columns, viz. $\left\{4^{3},\{\lambda\}_{A-16}\right\}$.

Finally, one obtains

$$
\begin{aligned}
\widehat{D}\{\lambda\}_{2 A-20}^{(A)} \stackrel{\circ}{=} & \sum_{\{\lambda\}_{A-16}} \alpha\left(\{2\} \dot{\otimes}\{\lambda\}_{A-16}\right\} \rightarrow\{\lambda\}_{2(A-16)}= \\
& \left.\{\lambda\}_{2 A-20}^{(A)}-\left\{4^{3}\right\}\right)\left\{4^{3},\{\lambda\}_{A-16}\right\} \quad(s-d)-\text { nuclei } .
\end{aligned}
$$

The Schur functions $\left\{4^{3},\{\lambda\}_{A-16}\right\}$ will produce, by Eq.(98), $S(A)$ irreps

$$
\left[A-(4 \times 3+A-16), 4^{3},\{\lambda\}_{A-16}\right]=\left[4^{4},\{\lambda\}_{A-16}\right] .
$$

Eqs.(145),(146) allow us to obtain the reduction $U(A-1) \supset S(A)$ for $(s-d)$-nuclei in ground configuration directly from the tables of multiplicities of Schur functions $\{\lambda\}_{2(A-16)}$ in the reduced plethysm $\{2\} \dot{\otimes}\{\lambda\}_{A-16}$. The

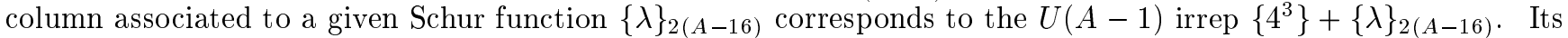
entries, in each line labelled by $\{2\} \otimes\{\lambda\}_{A-16}$, give the multiplicity of $S(A)$ irrep $\left[4^{4},\{\lambda\}_{A-16}\right]$ in the reduction.

As an example, for $A=19$, one has $E_{\min }=18, i_{\min }=i_{\max }=3$ and, from the plethysms $\{2\} \otimes\{\lambda\}_{3}$ given in table II, one obtains

$$
\begin{array}{ll}
\left\{10,4^{2}\right\} \stackrel{\circ}{=}\left[4^{4} 3\right] ; & \left\{7^{2} 4\right\} \stackrel{\circ}{=}\left[4^{4} 1^{3}\right] ; \\
\{954\} \stackrel{\circ}{=}\left[4^{4} 21\right] ; & \{765\} \stackrel{\circ}{=}\left[4^{4} 21\right] ; \\
\{864\} \stackrel{\circ}{=}\left[4^{4} 3\right]+\left[4^{4} 21\right] ; & \left\{6^{3}\right\} \stackrel{\circ}{=}\left[4^{4} 3\right] ; \\
\left\{85^{2}\right\} \stackrel{\circ}{=}\left[4^{4} 1^{3}\right] . &
\end{array}
$$

Making all reductions one observes that the results also present the symmetry under particle-hole exchange in the open shell [ Eqs.(138),( 139) ] that for this shell reads as

$$
\begin{aligned}
A & \rightarrow \bar{A}=16+(40-A)=56-A \\
{[\lambda] } & \equiv\left[4^{4}, \lambda_{1}^{(0)}, \lambda_{2}^{(0)}, \ldots, \lambda_{6}^{(0)}\right] \rightarrow[\bar{\lambda}]=\left[4^{4}, 4-\lambda_{6}^{(0)}, 4-\lambda_{5}^{(0)}, \ldots, 4-\lambda_{1}^{(0)}\right] .
\end{aligned}
$$

In Appendix B one gives the reduction $U(A-1) \supset S(A)$ for the first half of the shell. To obtain the reduction for the second half one uses again symmetry relations Eqs.(138),(139). The case $A=40$ is not covered by the symmetry . For $A=40$ only one $U(39)$ irrep has physically acceptable $A(40)$ irreps:

$$
\left\{20^{3}\right\} \stackrel{\circ}{=}\left[4^{10}\right] \text {. }
$$

For $(p-f)$-nuclei $(41 \leq A \leq 80), E_{0}=3$ and $E_{\min }=3 A-60$. One easily verifies that for those values of $A$ and $E$ one solution of Eqs.(132),(133) is

$$
t_{2}=24 ; \quad t_{3}=A-40 ; \quad t_{4}=t_{5}=0 ; \quad j_{2}=j_{3}=\ldots=0 .
$$

Computer calculations show that indeed this is the only solution. Therefore one has

$$
\widehat{D}\{\lambda\}_{3 A-60}^{(A)} \doteq \sum_{\{\lambda\}_{24}}\{\lambda\}_{24}\{\lambda\}_{A-40} \widehat{\mathcal{D}}\left(\{2\} \dot{\otimes}\{\lambda\}_{24}\right) \widehat{\mathcal{D}}\left(\{3\} \dot{\otimes}\{\lambda\}_{A-40}\right)\{\lambda\}_{3 A-60}^{(A)}
$$

and we need to compute, for the whole shell, only the reduced plethysms $\{3\} \dot{\otimes}\{\mu\}_{m}$ with $m=1,2, \ldots, 40$. As seen previously, only those reduced plethysms in which $\{\mu\}_{m}$ has up to 10 lines and 4 columns contribute.

Since only contribute Schur functions $\{\lambda\}_{24}$ with up to 6 lines and 4 columns, one has only one possible value, $\{\lambda\}_{24}=\left\{4^{6}\right\}$. Using this result and $\{2\} \dot{\otimes}\left\{4^{6}\right\}=\left\{16^{3}\right\}$, Eq.(151) becomes

$$
\widehat{D}\{\lambda\}_{3 A-60}^{(A)} \sum_{\{\lambda\}_{A-40}}\left\{4^{6},\{\lambda\}_{A-40}\right\} \widehat{\mathcal{D}}\left(\left\{16^{3}\right\}\right) \widehat{\mathcal{D}}\left(\{3\} \dot{\otimes}\{\lambda\}_{A-40}\right)\{\lambda\}_{3 A-60}^{(A)} .
$$

Now, linearizing the product of $\widehat{\mathcal{D}} \mathrm{s}$ and taking into account that only arguments of $\widehat{\mathcal{D}}$ with up to 3 rows contribute, one obtains 


$$
\begin{aligned}
& \widehat{D}\{\lambda\}_{3 A-60}^{(A)} \stackrel{\circ}{=} \sum_{\{\lambda\}_{A-40}}\left\{4^{6},\{\lambda\}_{A-40}\right\} \sum_{\{\lambda\}_{3(A-40)}} \alpha\left(\{3\} \dot{\otimes}\{\lambda\}_{A-40} \rightarrow\{\lambda\}_{3(A-40)}\right) . \\
& \widehat{\mathcal{D}}\left(\left\{16^{3}\right\}+\{\lambda\}_{3(A-40)}\right)\{\lambda\}_{3 A-60}^{(A)} .
\end{aligned}
$$

When acting on $\{\lambda\}_{3 A-60}^{\{(A)}$, the operator $\widehat{\mathcal{D}}\left(\left\{16^{3}\right\}+\{\lambda\}_{3 A-40}\right)$ will produce Schur functions $\{\nu\}$ with up to 3 lines and 4 columns of degree 12 what fixes $\{\nu\}=\left\{4^{3}\right\}$ and $\{\lambda\}_{3(A-40)}=\{\lambda\}_{3 A-60}-\left\{20^{3}\right\}$. Therefore one obtains the final result

$$
\begin{aligned}
\widehat{D}\{\lambda\}_{3 A-60}^{(A)} \stackrel{\circ}{ } & \sum_{\{\lambda\}_{A-40}} \alpha\left(\{3\} \dot{\otimes}\{\lambda\}_{A-40} \rightarrow\right. \\
& \left.\{\lambda\}_{3(A-40)}=\{\lambda\}_{3 A-60}-\left\{20^{3}\right\}\right)\left\{4^{9},\{\lambda\}_{A-40}\right\} .
\end{aligned}
$$

The Schur functions $\left\{4^{9},\{\lambda\}_{A-40}\right\}$ will produce, by Eq.( 98) $S(A)$ irreps

$$
\left[A-(4 \times 9+A-40), 4^{9},\{\lambda\}_{A-40}\right]=\left[4^{10},\{\lambda\}_{A-40}\right] .
$$

Analogously to case $E_{0}=1$ and 2 , Eqs.(154),(155) allow us to read the reduction $U(A-1) \supset S(A)$ for nuclei in the ground configuration of this shell directly form the table of multiplicities of Schur functions $\{\lambda\}_{3(A-40)}$ in the reduced plethysms $\{3\} \dot{\otimes}\{\lambda\}_{A-40}$. The column associated to a given Schur function $\{\lambda\}_{3(A-40)}$ corresponds to the $U(A-1)$ irrep $\left\{20^{3}\right\}+\{\lambda\}_{3(A-40)}$. Its entries, in each line labelled by $\{3\} \otimes\{\lambda\}_{A-40}$ give the multiplicity of $S(A)$ irrep $\left[4^{10},\{\lambda\}_{A-40}\right]$ in the reduction.

The results for the whole shell present the particle-hole exchange symmetry Eqs.(138),(139) that for this shell reads as

$$
\begin{aligned}
& A \leftrightarrow \bar{A}=120-A \\
& {[\lambda] \equiv\left[4^{10}, \lambda_{1}^{(0)}, \lambda_{2}^{(0)}, \ldots, \lambda_{10}^{(0)}\right] \rightarrow[\bar{\lambda}]=\left[4^{10}, 4-\lambda_{10}^{(0)}, 4-\lambda_{9}^{(0)}, \ldots, 4-\lambda_{1}^{(0)}\right] .}
\end{aligned}
$$

For $(s, d, g)$-nuclei $(81 \leq A \leq 140), E_{0}=4$ and $E_{\min }=4 A-140$. One easily verify that, for given $A$ and $E=E_{\min }$, one solution for Eqs.(132),133) is

$$
\begin{aligned}
& t_{2}=24 ; \quad t_{3}=40 ; \quad t_{4}=A-80 ; \quad t_{5}=t_{6}=\ldots=0 ; \\
& j_{2}=j_{3}=\ldots=0 .
\end{aligned}
$$

Again, computer calculations show that this is the only solution. Therefore one has

$$
\begin{aligned}
& \widehat{D}\{\lambda\}_{4 A-140}^{(A)} \stackrel{\circ}{=} \sum_{\{\lambda\}_{24}}\{\lambda\}_{24} \widehat{\mathcal{D}}\left(\{2\} \dot{\otimes}\{\lambda\}_{24}\right) \sum_{\{\lambda\}_{40}}\{\lambda\}_{40} \widehat{\mathcal{D}}\left(\{3\} \dot{\otimes}\left\{\{\lambda\}_{40}\right) .\right. \\
& \sum_{\{\lambda\}_{A-80}}\{\lambda\}_{A-80} \widehat{\mathcal{D}}\left(\{4\} \dot{\otimes}\{\lambda\}_{A-80}\right)\{\lambda\}_{4 A-140}^{(A)} .
\end{aligned}
$$

Using arguments similar to those used in cases $E_{0}=2,3$ and the result $\{3\} \dot{\otimes}\left\{4^{10}\right\}=\left\{40^{3}\right\}$ one deduces that

$$
\begin{aligned}
\widehat{D}\{\lambda\}_{4 A-140}^{(A)} \doteq & \sum_{\{\lambda\}_{A-80}}\left\{4^{19},\{\lambda\}_{A-80}\right\} \alpha\left(\{4\} \dot{\otimes}\{\lambda\}_{A-80} \rightarrow\{\lambda\}_{4(A-80)}=\right. \\
& \left.\{\lambda\}_{4 A-140}^{(A)}-\left\{60^{3}\right\}\right)
\end{aligned}
$$

and the Schur functions $\left\{4^{19},\{\lambda\}_{A-80}\right\}$ will produce $S(A)$ irreps $\left[4^{20},\{\lambda\}_{A-80}\right]$.

As seen in the previous cases, the reduction $U(A-1) \supset S(A)$ can be read from the table of multiplicities os Schur functions $\{\lambda\}_{4(A-80)}$ in the reduced plethysm $\{4\} \dot{\otimes}\{\lambda\}_{A-80}$. The column of $\{\lambda\}_{4(A-80)}$ is associated to the $U(A-1)$ irrep $\left\{60^{3}\right\}+\{\lambda\}_{4(A-80)}$. Its entries give the multiplicities of $\left[4^{20},\{\lambda\}_{A-80}\right]$ in the reduction.

From the cases studied, one pattern emerges:

1) The $t_{k}$ 's and $j_{k}$ 's that contribute are 


$$
t_{k}=2(k+1)(k+2) ; \quad k=2,3, \ldots, E_{0}-1 ; t_{E_{0}}=n_{0} \equiv A-n_{\text {core }} ;
$$

2)The final result is

$$
\begin{aligned}
\widehat{D}\{\lambda\}_{E_{\text {min }}}^{(A)} \stackrel{\circ}{=} & \sum_{\{\lambda\}_{n_{0}}}\left\{4^{n_{\text {core }} / 4-1},\{\lambda\}_{n_{0}}\right\} \alpha\left(\left\{E_{0}\right\} \dot{\otimes}\{\lambda\}_{n_{0}} \rightarrow\{\lambda\}_{E_{0} n_{0}}=\right. \\
& \left.\{\lambda\}_{E_{\text {min }}}^{(A)}-\left\{\left(n_{\text {core }}-2 E_{0}\left(E_{0}+1\right)\right)^{3}\right\}\right)
\end{aligned}
$$

and the Schur functions $\left\{4^{n_{\text {core }} / 4-1},\{\lambda\}_{n_{0}}\right\}$ will produce $S(A)$ ireps $\left[4^{n_{\text {core }} / 4},\{\lambda\}_{n_{0}}\right]$.

\section{Final Comments}

According to the subgroup chain Eq.(89) we need to classify the basis states by the chain $U^{(r)}(A-1) \supset$ $O^{(r)}(A-1) \supset S^{(r)}(A)$ but up to now we obtained the reduction by the chain $U^{(r)}(A-1) \supset S^{(r)}(A)$.

For configuration energies $E=E_{\text {min }}$ and $E_{\text {min }}+1$, a given $U^{(r)}(A-1)$ irrep $\left\{E_{1}, E_{2}, E_{3}\right\}$ gives only one $O^{(r)}(A-1)$ irrep $(\omega)=\left(E_{1}, E_{2}, E_{3}\right)$. Therefore our classification of ground state configuration states is complete.

For configuration energies $E \geq E_{\text {min }}+2$ one needs first to express, the $O^{(r)}(A-1)$ irrep $(\omega)$ in terms of $U^{(r)}(A-1)$ irreps $\left\{E^{\prime}\right\}$ and then perform the reductions $\left\{E^{\prime}\right\} \rightarrow[f]$.

In application to a definite nucleus with $Z$ protons and $N=A-Z$ neutrons, the isospin counterpart imposes one selection rule

$$
\frac{k_{3}+2 k_{2}+k_{1}}{2} \geq\left|T_{Z}\right|=\left|\frac{Z-N}{2}\right|
$$

that may forbid some configurations in the reduction.

\section{Appendix A. Classification of the Lowest Energy States for $p$-Nuclei}

This classification was obtained using Eqs.(136) and (137). In the third column we have put the $S U(3)$ labels in Elliott notation: $p=E_{1}-E_{2}, q=E_{2}-E_{3}$. Observe that there is a symmetry under the interchange of particles and holes in the open $p$ shell, that is, the labels associated to $A$ are related to those of $\bar{A}=4+(16-A)=20-A$. For the $S U(3)$ labels the association is $\bar{p}=q, \bar{q}=p$. For the $S(A)$ labels, writing $[\lambda]=\left[4, \lambda_{1}^{(0)}, \lambda_{2}^{(0)}, \lambda_{3}^{(0)}\right]$, the $S(\bar{A})$ become $[\bar{\lambda}]=\left[4,4-\lambda_{3}^{(0)}, 4-\lambda_{2}^{(0)}, 4-\lambda_{1}^{(0)}\right]$.

\begin{tabular}{|c|c|c|c|}
\hline $\mathrm{A}$ & $\{\mathrm{E}\}$ & $(\mathrm{pq})$ & {$[\lambda]$} \\
\hline 5 & $\{1\}$ & $(10)$ & {$[41]$} \\
\hline 6 & $\{2\}$ & $(20)$ & {$[42]$} \\
& $\left\{1^{2}\right\}$ & $(01)$ & {$\left[41^{2}\right]$} \\
\hline 7 & $\{3\}$ & $(30)$ & {$[43]$} \\
& $\{21\}$ & $(11)$ & {$[421]$} \\
& $\left\{1^{3}\right\}$ & $(00)$ & {$\left[41^{3}\right]$} \\
\hline 8 & $\{4\}$ & $(40)$ & {$\left[4^{2}\right]$} \\
& $\{31\}$ & $(21)$ & {$[431]$} \\
& $\left\{2^{2}\right\}$ & $(02)$ & {$\left[42^{2}\right]$} \\
& $\left\{21^{2}\right\}$ & $(10)$ & {$\left[421^{2}\right]$} \\
\hline 9 & $\{41\}$ & $(31)$ & {$\left[4^{2} 1\right]$} \\
& $\{32\}$ & $(12)$ & {$[432]$} \\
& $\left\{31^{2}\right\}$ & $(20)$ & {$\left[431^{2}\right]$} \\
& $\left\{2^{2} 1\right\}$ & $(01)$ & {$\left[42^{2} 1\right]$} \\
\hline 10 & $\{42\}$ & $(22)$ & {$\left[4^{2} 2\right]$} \\
& $\left\{41^{2}\right\}$ & $(30)$ & {$\left[4^{2} 1^{2}\right]$} \\
& $\left\{3^{2}\right\}$ & $(03)$ & {$\left[43^{2}\right]$} \\
& $\{321\}$ & $(11)$ & {$[4321]$} \\
& $\left\{2^{3}\right\}$ & $(00)$ & {$\left[42^{3}\right]$} \\
\hline
\end{tabular}

\begin{tabular}{|c|c|c|c|}
\hline $\mathrm{A}$ & $\{\mathrm{E}\}$ & $(\mathrm{pq})$ & {$[\lambda]$} \\
\hline 11 & $\{43\}$ & $(13)$ & {$\left[4^{2} 3\right]$} \\
& $\{421\}$ & $(21)$ & {$\left[4^{2} 21\right]$} \\
& $\left\{3^{2} 1\right\}$ & $(02)$ & {$\left[43^{2} 1\right]$} \\
& $\left\{32^{2}\right\}$ & $(10)$ & {$\left[432^{2}\right]$} \\
\hline 12 & $\left\{4^{2}\right\}$ & $(04)$ & {$\left[4^{3}\right]$} \\
& $\{431\}$ & $(12)$ & {$\left[4^{2} 31\right]$} \\
& $\left\{42^{2}\right\}$ & $(20)$ & {$\left[4^{2} 2^{2}\right]$} \\
& $\left\{3^{2} 2\right\}$ & $(01)$ & {$\left[43^{2} 2\right]$} \\
\hline 13 & $\left\{4^{2} 1\right\}$ & $(03)$ & {$\left[4^{3} 1\right]$} \\
& $\{432\}$ & $(11)$ & {$\left[4^{2} 32\right]$} \\
& $\left\{3^{3}\right\}$ & $(00)$ & {$\left[43^{3}\right]$} \\
\hline 14 & $\left\{4^{2} 2\right\}$ & $(02)$ & {$\left[4^{3} 2\right]$} \\
& $\left\{43^{2}\right\}$ & $(10)$ & {$\left[4^{2} 3^{2}\right]$} \\
\hline 15 & $\left\{4^{2} 3\right\}$ & $(01)$ & {$\left[4^{3} 3\right]$} \\
\hline 16 & $\left\{4^{3}\right\}$ & $(00)$ & {$\left[4^{4}\right]$} \\
\hline
\end{tabular}


Appendix B. Classification of the Lowest Energy States for $(s-d)$-Nuclei $s-d(17 \leq A \leq 40)$

This classification was obtained from the table of plethysms $\{2\} \dot{\otimes}\{\lambda\}_{i_{\max }}$ with $i_{\max }=A-16$, according to the text in Section 4. For the second half of the shell we used the particle-hole symmetry for this shell. By it, to a given $A$ we associate a $\bar{A}=16+(40-A)=56-A$. Again, for the $S U(3)$ labels this simmetry gives $\bar{p}=q$ and $\bar{q}=p$. To the $S(A)$ label $[\lambda]=\left[4^{4}, \lambda_{1}^{(0)}, \lambda_{2}^{(0)}, \ldots, \lambda_{6}^{(0)}\right]$ we associate the $S(\bar{A})$ label $[\bar{\lambda}]=\left[4-\lambda_{6}^{(0)}, 4-\lambda_{5}^{(0)}, \ldots, 4-\lambda_{1}^{(0)}\right]$.

$$
\begin{aligned}
& \mathrm{A}=17 \\
& \left\{6,4^{2}\right\} \stackrel{\circ}{=}\left[4^{4} 1\right] \\
& \mathrm{A}=18 \\
& \left\{8,4^{2}\right\} \stackrel{\circ}{\circ}\left[4^{4} 2\right] \\
& \{7,5,4\} \stackrel{\circ}{=}\left[4^{4} 1^{2}\right] \\
& \left\{6^{2}, 4\right\} \stackrel{\circ}{=}\left[4^{4} 2\right] \\
& \mathrm{A}=19 \\
& \left\{10,4^{2}\right\} \stackrel{\circ}{\circ}\left[4^{4} 3\right] \\
& \{9,5,4\} \stackrel{\circ}{=}\left[4^{4} 21\right] \\
& \{8,6,4\} \stackrel{\circ}{=}\left[4^{4} 21\right]+\left[4^{4} 3\right] \\
& \left\{8,5^{2}\right\} \stackrel{\circ}{\circ}\left[4^{4} 1^{3}\right] \\
& \left\{7^{2}, 4\right\} \stackrel{\circ}{=}\left[4^{4} 1^{3}\right] \\
& \{7,6,5\} \stackrel{\circ}{=}\left[4^{4} 21\right] \\
& \left\{6^{3}\right\} \stackrel{\circ}{=}\left[4^{4} 3\right] \\
& \mathrm{A}=20 \\
& \left\{12,4^{2}\right\} \stackrel{\circ}{=}\left[4^{5}\right] \\
& \{11,5,4\} \stackrel{\circ}{=}\left[4^{4} 31\right] \\
& \{10,6,4\} \stackrel{\circ}{=}\left[4^{4} 2^{2}\right]+\left[4^{4} 31\right]+\left[4^{5}\right] \\
& \left\{10,5^{2}\right\} \stackrel{\circ}{=}\left[4^{4} 21^{2}\right] \\
& \{9,7,4\} \stackrel{\circ}{=}\left[4^{4} 21^{2}\right]+\left[4^{4} 31\right] \\
& \left\{8^{2}, 4\right\} \stackrel{\circ}{\circ}\left[4^{4} 2^{2}\right]+\left[4^{5}\right] \\
& \{9,6,5\} \stackrel{\circ}{=}\left[4^{4} 21^{2}\right]+\left[4^{4} 2^{2}\right]+\left[4^{4} 31\right] \\
& \{8,7,5\} \stackrel{\circ}{=}\left[4^{4} 1^{4}\right]+\left[4^{4} 21^{2}\right]+\left[4^{4} 31\right] \\
& \left\{8,6^{2}\right\} \stackrel{\circ}{\circ}\left[4^{4} 2^{2}\right]+\left[4^{4} 31\right]+\left[4^{5}\right] \\
& \left\{7^{2}, 6\right\} \stackrel{\circ}{=}\left[4^{4} 21^{2}\right]
\end{aligned}
$$

\section{$\mathrm{A}=21$}

$\{13,5,4\} \stackrel{\circ}{=}\left[4^{5} 1\right]$

$\{12,6,4\} \stackrel{\circ}{=}\left[4^{4} 32\right]+\left[4^{5} 1\right]$

$\left\{12,5^{2}\right\} \stackrel{\circ}{=}\left[4^{4} 31^{2}\right]$

$\{11,7,4\} \stackrel{\circ}{=}\left[4^{4} 31^{2}\right]+\left[4^{4} 32\right]+\left[4^{5} 1\right]$

$\{11,6,5\} \stackrel{\circ}{=}\left[4^{4} 2^{2} 1\right]+\left[4^{4} 31^{2}\right]+\left[4^{4} 32\right]+\left[4^{5} 1\right]$

$\{10,8,4\} \stackrel{\circ}{=}\left[4^{4} 2^{2} 1\right]+\left[4^{4} 32\right]+\left[4^{5} 1\right]$

$\left\{9^{2}, 4\right\} \stackrel{\circ}{=}\left[4^{4} 31^{2}\right]$

$\{10,7,5\} \stackrel{\circ}{=}\left[4^{4} 21^{3}\right]+\left[4^{4} 2^{2} 1\right]+2\left[4^{4} 31^{2}\right]+\left[4^{4} 32\right]+\left[4^{5} 1\right]$

$\left\{10,6^{2}\right\} \stackrel{\circ}{=}\left[4^{4} 2^{2} 1\right]+2\left[4^{4} 32\right]+\left[4^{5} 1\right]$

$\{9,8,5\} \stackrel{\circ}{=}\left[4^{4} 21^{3}\right]+\left[4^{4} 2^{2} 1\right]+\left[4^{4} 31^{2}\right]+\left[4^{4} 32\right]+\left[4^{5} 1\right]$

$\{9,7,6\} \stackrel{\circ}{=}\left[4^{4} 21^{3}\right]+\left[4^{4} 2^{2} 1\right]+2\left[4^{4} 31^{2}\right]+\left[4^{4} 32\right]+\left[4^{5} 1\right]$

$\left\{8^{2}, 6\right\} \stackrel{\circ}{=}\left[4^{4} 1^{5}\right]+\left[4^{4} 2^{2} 1\right]+\left[4^{4} 32\right]+\left[4^{5} 1\right]$

$\left\{8,7^{2}\right\} \stackrel{\circ}{=}\left[4^{4} 21^{3}\right]+\left[4^{4} 31^{2}\right]$

$\mathrm{A}=22$ 


$$
\begin{aligned}
& \{14,6,4\} \stackrel{\circ}{\circ}\left[4^{5} 2\right] \\
& \left\{14,5^{2}\right\} \stackrel{\circ}{=}\left[4^{5} 1^{2}\right] \\
& \{13,7,4\} \stackrel{\circ}{=}\left[4^{5} 1^{2}\right]+\left[4^{4} 3^{2}\right]+\left[4^{5} 2\right] \\
& \{13,6,5\} \stackrel{\circ}{\circ}\left[4^{4} 321\right]+\left[4^{5} 1^{2}\right]+\left[4^{5} 2\right] \\
& \{12,8,4\} \stackrel{\circ}{\circ}\left[4^{4} 321\right]+2\left[4^{5} 2\right] \\
& \{12,7,5\} \stackrel{\circ}{=}\left[4^{4} 31^{3}\right]+2\left[4^{4} 321\right]+2\left[4^{5} 1^{2}\right]+\left[4^{4} 3^{2}\right]+\left[4^{5} 2\right] \\
& \{11,9,4\} \stackrel{\circ}{\circ}\left[4^{4} 321\right]+\left[4^{5} 1^{2}\right]+\left[4^{4} 3^{2}\right] \\
& \{12,6\}=\left[6^{2}\right\} \stackrel{\circ}{=}\left[4^{4} 2^{3}\right]+\left[4^{4} 321\right]+\left[4^{4} 3^{2}\right]+2\left[4^{5} 2\right] \\
& \left\{10^{2}, 4\right\} \stackrel{\circ}{=}\left[4^{4} 2^{3}\right]+\left[4^{5} 2\right] \\
& \{11,8,5\} \stackrel{\circ}{=}\left[4^{4} 2^{2} 1^{2}\right]+\left[4^{4} 31^{3}\right]+\left[4^{4} 2^{3}\right]+2\left[4^{4} 321\right]+2\left[4^{5} 1^{2}\right]+\left[4^{4} 3^{2}\right]+2\left[4^{5} 2\right] \\
& \{11,7,6\} \stackrel{\circ}{=}\left[4^{4} 2^{2} 1^{2}\right]+\left[4^{4} 31^{3}\right]+3\left[4^{4} 321\right]+2\left[4^{5} 1^{2}\right]+\left[4^{4} 3^{2}\right]+2\left[4^{5} 2\right] \\
& \{10,9,5\} \stackrel{\circ}{=}\left[4^{4} 2^{2} 1^{2}\right]+\left[4^{4} 31^{3}\right]+2\left[4^{4} 321\right]+\left[4^{5} 1^{2}\right]+\left[4^{5} 2\right] \\
& \{10,8,6\} \stackrel{\circ}{=}\left[4^{4} 21^{4}\right]+\left[4^{4} 2^{2} 1^{2}\right]+\left[4^{4} 31^{3}\right]+2\left[4^{4} 2^{3}\right]+3\left[4^{4} 321\right]+\left[4^{5} 1^{2}\right]+\left[4^{4} 3^{2}\right]+
\end{aligned}
$$
$3\left[4^{5} 2\right]$

$\left\{10,7^{2}\right\} \stackrel{\circ}{=}\left[4^{4} 2^{2} 1^{2}\right]+2\left[4^{4} 31^{3}\right]+\left[4^{4} 321\right]+2\left[4^{5} 1^{2}\right]+\left[4^{4} 3^{2}\right]$

$\left\{9^{2}, 6\right\} \stackrel{\circ}{=}\left[4^{4} 2^{2} 1^{2}\right]+\left[4^{4} 31^{3}\right]+\left[4^{4} 321\right]+2\left[4^{5} 1^{2}\right]+\left[4^{4} 3^{2}\right]$

$\{9,8,7\} \stackrel{\circ}{=}\left[4^{4} 21^{4}\right]+\left[4^{4} 2^{2} 1^{2}\right]+\left[4^{4} 31^{3}\right]+2\left[4^{4} 321\right]+\left[4^{5} 1^{2}\right]+\left[4^{5} 2\right]$

$\left\{8^{3}\right\} \stackrel{\circ}{=}\left[4^{4} 1^{6}\right]+\left[4^{4} 2^{3}\right]+\left[4^{5} 2\right]$

$$
\begin{aligned}
& \mathrm{A}=23 \\
& \{15,7,4\} \stackrel{\circ}{=}\left[4^{5} 3\right] \\
& \{15,6,5\} \stackrel{\circ}{=}\left[4^{5} 21\right] \\
& \{14,8,4\} \stackrel{\circ}{=}\left[4^{5} 21\right]+\left[4^{5} 3\right] \\
& \{14,7,5\} \stackrel{\circ}{=}\left[4^{5} 1^{3}\right]+\left[4^{4} 3^{2} 1\right]+2\left[4^{5} 21\right]+\left[4^{5} 3\right] \\
& \left\{14,6^{2}\right\} \stackrel{\circ}{=}\left[4^{4} 32^{2}\right]+\left[4^{5} 21\right]+\left[4^{5} 3\right] \\
& \{13,9,4\} \stackrel{\circ}{=}\left[4^{4} 3^{2} 1\right]+\left[4^{5} 21\right]+\left[4^{5} 3\right] \\
& \{12,10,4\} \stackrel{\circ}{=}\left[4^{4} 32^{2}\right]+\left[4^{5} 21\right]+\left[4^{5} 3\right] \\
& \{13,8,5\} \stackrel{\circ}{=}\left[4^{4} 321^{2}\right]+\left[4^{5} 1^{3}\right]+\left[4^{4} 32^{2}\right]+\left[4^{4} 3^{2} 1\right]+3\left[4^{5} 21\right]+2\left[4^{5} 3\right] \\
& \left\{11^{2}, 4\right\} \stackrel{\circ}{=}\left[4^{4} 3^{2} 1\right] \\
& \{13,7,6\} \stackrel{\circ}{=}\left[4^{4} 321^{2}\right]+\left[4^{5} 1^{3}\right]+\left[4^{4} 32^{2}\right]+2\left[4^{4} 3^{2} 1\right]+3\left[4^{5} 21\right]+2\left[4^{5} 3\right] \\
& \{12,9,5\} \stackrel{\circ}{\circ} 2\left[4^{4} 321^{2}\right]+\left[4^{5} 1^{3}\right]+\left[4^{4} 32^{2}\right]+2\left[4^{4} 3^{2} 1\right]+3\left[4^{5} 21\right]+\left[4^{5} 3\right] \\
& \{11,10,5\} \stackrel{\circ}{=}\left[4^{4} 2^{3} 1\right]+\left[4^{4} 321^{2}\right]+\left[4^{5} 1^{3}\right]+\left[4^{4} 32^{2}\right]+\left[4^{4} 3^{2} 1\right]+2\left[4^{5} 21\right]+\left[4^{5} 3\right] \\
& \{12,8,6\} \stackrel{\circ}{=}\left[4^{4} 31^{4}\right]+\left[4^{4} 2^{3} 1\right]+2\left[4^{4} 321^{2}\right]+\left[4^{5} 1^{3}\right]+3\left[4^{4} 32^{2}\right]+2\left[4^{4} 3^{2} 1\right]+ \\
& 5\left[4^{5} 21\right]+3\left[4^{5} 3\right] \\
& \left\{12,7^{2}\right\} \stackrel{\circ}{=} 2\left[4^{4} 321^{2}\right]+2\left[4^{5} 1^{3}\right]+2\left[4^{4} 3^{2} 1\right]+2\left[4^{5} 21\right]+\left[4^{5} 3\right] \\
& \{11,9,6\} \stackrel{\circ}{=}\left[4^{4} 2^{2} 1^{3}\right]+\left[4^{4} 2^{3} 1\right]+3\left[4^{4} 321^{2}\right]+2\left[4^{5} 1^{3}\right]+2\left[4^{4} 32^{2}\right]+3\left[4^{4} 3^{2} 1\right]+ \\
& 4\left[4^{5} 21\right]+2\left[4^{5} 3\right] \\
& \left\{10^{2}, 6\right\} \stackrel{\circ}{=}\left[4^{4} 31^{4}\right]+\left[4^{4} 2^{3} 1\right]+\left[4^{4} 321^{2}\right]+2\left[4^{4} 32^{2}\right]+2\left[4^{5} 21\right]+\left[4^{5} 3\right] \\
& \{11,8,7\} \stackrel{\circ}{=}\left[4^{4} 2^{2} 1^{3}\right]+\left[4^{4} 31^{4}\right]+\left[4^{4} 2^{3} 1\right]+3\left[4^{4} 321^{2}\right]+2\left[4^{5} 1^{3}\right]+2\left[4^{4} 32^{2}\right]+ \\
& 2\left[4^{4} 3^{2} 1\right]+4\left[4^{5} 21\right]+2\left[4^{5} 3\right] \\
& \{10,9,7\} \stackrel{\circ}{=}\left[4^{4} 2^{2} 1^{3}\right]+\left[4^{4} 31^{4}\right]+\left[4^{4} 2^{3} 1\right]+3\left[4^{4} 321^{2}\right]+2\left[4^{5} 1^{3}\right]+\left[4^{4} 32^{2}\right]+ \\
& 2\left[4^{4} 3^{2} 1\right]+3\left[4^{5} 21\right]+\left[4^{5} 3\right] \\
& \left\{10,8^{2}\right\} \stackrel{\circ}{=}\left[4^{4} 21^{5}\right]+\left[4^{4} 31^{4}\right]+\left[4^{4} 2^{3} 1\right]+\left[4^{4} 321^{2}\right]+2\left[4^{4} 32^{2}\right]+2\left[4^{5} 21\right]+\left[4^{5} 3\right] \\
& \left\{9^{2}, 8\right\} \stackrel{\circ}{=}\left[4^{4} 2^{2} 1^{3}\right]+\left[4^{4} 321^{2}\right]+\left[4^{5} 1^{3}\right]+\left[4^{4} 3^{2} 1\right]+\left[4^{5} 21\right]
\end{aligned}
$$

\section{$\mathrm{A}=24$}

$\{16,8,4\} \stackrel{\circ}{=}\left[4^{6}\right]$

$\{16,7,5\} \stackrel{\circ}{=}\left[4^{5} 31\right]$

$\left\{16,6^{2}\right\} \stackrel{\circ}{=}\left[4^{5} 2^{2}\right]$

$\{15,9,4\} \stackrel{\circ}{=}\left[4^{5} 31\right]$

$\{15,8,5\} \stackrel{\circ}{=}\left[4^{5} 21^{2}\right]+\left[4^{5} 2^{2}\right]+2\left[4^{5} 31\right]+\left[4^{6}\right]$

$\{14,10,4\} \stackrel{\circ}{=}\left[4^{5} 2^{2}\right]+\left[4^{5} 31\right]+\left[4^{6}\right]$

$\{15,7,6\} \stackrel{\circ}{=}\left[4^{5} 21^{2}\right]+\left[4^{4} 3^{2} 2\right]+\left[4^{5} 2^{2}\right]+2\left[4^{5} 31\right]+\left[4^{6}\right]$

$\{13,11,4\} \stackrel{\circ}{=}\left[4^{4} 3^{2} 2\right]+\left[4^{5} 31\right]$

$\{14,9,5\} \stackrel{\circ}{=}\left[4^{4} 3^{2} 1^{2}\right]+2\left[4^{5} 21^{2}\right]+\left[4^{4} 3^{2} 2\right]+\left[4^{5} 2^{2}\right]+3\left[4^{5} 31\right]+\left[4^{6}\right]$

$\left\{12^{2}, 4\right\} \stackrel{\circ}{=}\left[4^{5} 2^{2}\right]+\left[4^{6}\right]$

$\{14,8,6\} \stackrel{\circ}{=}\left[4^{5} 1^{4}\right]+\left[4^{4} 32^{2} 1\right]+\left[4^{4} 3^{2} 1^{2}\right]+2\left[4^{5} 21^{2}\right]+\left[4^{4} 3^{2} 2\right]+4\left[4^{5} 2^{2}\right]+$ 
$4\left[4^{5} 31\right]+2\left[4^{6}\right]$

$\{13,10,5\} \stackrel{\circ}{=}\left[4^{4} 32^{2} 1\right]+\left[4^{4} 3^{2} 1^{2}\right]+2\left[4^{5} 21^{2}\right]+\left[4^{4} 3^{2} 2\right]+2\left[4^{5} 2^{2}\right]+3\left[4^{5} 31\right]+\left[4^{6}\right]$

$\left\{14,7^{2}\right\} \stackrel{\circ}{=}\left[4^{4} 3^{2} 1^{2}\right]+2\left[4^{5} 21^{2}\right]+\left[4^{4} 3^{2} 2\right]+3\left[4^{5} 31\right]$

$\{12,11,5\} \stackrel{\circ}{=}\left[4^{4} 32^{2} 1\right]+\left[4^{4} 3^{2} 1^{2}\right]+\left[4^{5} 21^{2}\right]+\left[4^{4} 3^{2} 2\right]+\left[4^{5} 2^{2}\right]+2\left[4^{5} 31\right]$

$\{13,9,6\} \stackrel{\circ}{=}\left[4^{4} 321^{3}\right]+2\left[4^{4} 32^{2} 1\right]+2\left[4^{4} 3^{2} 1^{2}\right]+4\left[4^{5} 21^{2}\right]+3\left[4^{4} 3^{2} 2\right]+3\left[4^{5} 2^{2}\right]+$ $6\left[4^{5} 31\right]+\left[4^{6}\right]$

$\{13,8,7\} \stackrel{\circ}{=}\left[4^{4} 321^{3}\right]+\left[4^{5} 1^{4}\right]+2\left[4^{4} 32^{2} 1\right]+2\left[4^{4} 3^{2} 1^{2}\right]+4\left[4^{5} 21^{2}\right]+2\left[4^{4} 3^{2} 2\right]+$ $3\left[4^{5} 2^{2}\right]+5\left[4^{5} 31\right]+2\left[4^{6}\right]$

$\{12,10,6\} \stackrel{\circ}{=}\left[4^{4} 321^{3}\right]+\left[4^{5} 1^{4}\right]+\left[4^{4} 2^{4}\right]+3\left[4^{4} 32^{2} 1\right]+2\left[4^{4} 3^{2} 1^{2}\right]+3\left[4^{5} 21^{2}\right]+$ $2\left[4^{4} 3^{2} 2\right]+5\left[4^{5} 2^{2}\right]+4\left[4^{5} 31\right]+2\left[4^{6}\right]$

$\left\{11^{2}, 6\right\} \stackrel{\circ}{=}\left[4^{4} 2^{3} 1^{2}\right]+\left[4^{4} 32^{2} 1\right]+\left[4^{4} 3^{2} 1^{2}\right]+2\left[4^{5} 21^{2}\right]+2\left[4^{4} 3^{2} 2\right]+2\left[4^{5} 31\right]$

$\{12,9,7\} \stackrel{\circ}{=}\left[4^{4} 2^{3} 1^{2}\right]+2\left[4^{4} 321^{3}\right]+\left[4^{5} 1^{4}\right]+3\left[4^{4} 32^{2} 1\right]+4\left[4^{4} 3^{2} 1^{2}\right]+6\left[4^{5} 21^{2}\right]+$ $3\left[4^{4} 3^{2} 2\right]+3\left[4^{5} 2^{2}\right]+6\left[4^{5} 31\right]+\left[4^{6}\right]$

$\left\{12,8^{2}\right\} \stackrel{\circ}{=}\left[4^{4} 31^{5}\right]+\left[4^{4} 321^{3}\right]+\left[4^{5} 1^{4}\right]+\left[4^{4} 2^{4}\right]+2\left[4^{4} 32^{2} 1\right]+\left[4^{4} 3^{2} 1^{2}\right]+$

$2\left[4^{5} 21^{2}\right]+\left[4^{4} 3^{2} 2\right]+4\left[4^{5} 2^{2}\right]+2\left[4^{5} 31\right]+2\left[4^{6}\right]$

$\{11,10,7\} \stackrel{\circ}{=}\left[4^{4} 2^{3} 1^{2}\right]+2\left[4^{4} 321^{3}\right]+\left[4^{5} 1^{4}\right]+\left[4^{4} 2^{4}\right]+3\left[4^{4} 32^{2} 1\right]+2\left[4^{4} 3^{2} 1^{2}\right]+$ $4\left[4^{5} 21^{2}\right]+2\left[4^{4} 3^{2} 2\right]+3\left[4^{5} 2^{2}\right]+4\left[4^{5} 31\right]+\left[4^{6}\right]$

$\{11,9,8\} \stackrel{\circ}{=}\left[4^{4} 2^{2} 1^{4}\right]+\left[4^{4} 2^{3} 1^{2}\right]+2\left[4^{4} 321^{3}\right]+\left[4^{5} 1^{4}\right]+3\left[4^{4} 32^{2} 1\right]+2\left[4^{4} 3^{2} 1^{2}\right]+$ $4\left[4^{5} 21^{2}\right]+2\left[4^{4} 3^{2} 2\right]+2\left[4^{5} 2^{2}\right]+4\left[4^{5} 31\right]$

$\left\{10^{2}, 8\right\} \stackrel{\circ}{=}\left[4^{4} 31^{5}\right]+\left[4^{4} 321^{3}\right]+\left[4^{5} 1^{4}\right]+\left[4^{4} 2^{4}\right]+2\left[4^{4} 32^{2} 1\right]+\left[4^{4} 3^{2} 1^{2}\right]+\left[4^{5} 21^{2}\right]+$ $3\left[4^{5} 2^{2}\right]+\left[4^{5} 31\right]+\left[4^{6}\right]$

$\left\{10,9^{2}\right\} \stackrel{\circ}{=}\left[4^{4} 2^{3} 1^{2}\right]+\left[4^{4} 321^{3}\right]+\left[4^{4} 3^{2} 1^{2}\right]+2\left[4^{5} 21^{2}\right]+\left[4^{4} 3^{2} 2\right]+\left[4^{5} 31\right]$

$\mathrm{A}=25$

$\{17,8,5\} \stackrel{\circ}{=}\left[4^{6} 1\right]$

$\{17,7,6\} \stackrel{\circ}{=}\left[4^{5} 32\right]$

$\{16,10,4\} \stackrel{\circ}{=}\left[4^{6} 1\right]$

$\{16,9,5\} \stackrel{\circ}{=}\left[4^{5} 31^{2}\right]+\left[4^{5} 32\right]+\left[4^{6} 1\right]$

$\{15,11,4\} \stackrel{\circ}{=}\left[4^{5} 32\right]$

$\{16,8,6\} \stackrel{\circ}{=}\left[4^{5} 2^{2} 1\right]+\left[4^{5} 31^{2}\right]+2\left[4^{5} 32\right]+2\left[4^{6} 1\right]$

$\{14,12,4\} \stackrel{\circ}{=}\left[4^{5} 32\right]+\left[4^{6} 1\right]$

$\left\{16,7^{2}\right\} \stackrel{\circ}{=}\left[4^{5} 31^{2}\right]+\left[4^{4} 3^{3}\right]+\left[4^{5} 32\right]+\left[4^{6} 1\right]$

$\left\{13^{2}, 4\right\} \stackrel{\circ}{=}\left[4^{4} 3^{3}\right]$

$\{15,10,5\} \stackrel{\circ}{=}\left[4^{5} 2^{2} 1\right]+2\left[4^{5} 31^{2}\right]+2\left[4^{5} 32\right]+2\left[4^{6} 1\right]$

$\{15,9,6\} \stackrel{\circ}{=}\left[4^{5} 21^{3}\right]+\left[4^{4} 3^{2} 21\right]+2\left[4^{5} 2^{2} 1\right]+3\left[4^{5} 31^{2}\right]+\left[4^{4} 3^{3}\right]+4\left[4^{5} 32\right]+3\left[4^{6} 1\right]$

$\{14,11,5\} \stackrel{\circ}{=}\left[4^{4} 3^{2} 21\right]+\left[4^{5} 2^{2} 1\right]+2\left[4^{5} 31^{2}\right]+\left[4^{4} 3^{3}\right]+2\left[4^{5} 32\right]+2\left[4^{6} 1\right]$

$\{15,8,7\} \stackrel{\circ}{=}\left[4^{5} 21^{3}\right]+\left[4^{4} 3^{2} 21\right]+2\left[4^{5} 2^{2} 1\right]+3\left[4^{5} 31^{2}\right]+4\left[4^{5} 32\right]+3\left[4^{6} 1\right]$

$\{13,12,5\} \stackrel{\circ}{=}\left[4^{4} 3^{2} 21\right]+\left[4^{5} 2^{2} 1\right]+\left[4^{5} 31^{2}\right]+2\left[4^{5} 32\right]+\left[4^{6} 1\right]$

$\{14,10,6\} \stackrel{\circ}{=}\left[4^{4} 3^{2} 1^{3}\right]+\left[4^{5} 21^{3}\right]+\left[4^{4} 32^{3}\right]+2\left[4^{4} 3^{2} 21\right]+4\left[4^{5} 2^{2} 1\right]+4\left[4^{5} 31^{2}\right]+$ $6\left[4^{5} 32\right]+4\left[4^{6} 1\right]$

$\{14,9,7\} \stackrel{\circ}{=}\left[4^{4} 32^{2} 1^{2}\right]+\left[4^{4} 3^{2} 1^{3}\right]+2\left[4^{5} 21^{3}\right]+3\left[4^{4} 3^{2} 21\right]+4\left[4^{5} 2^{2} 1\right]+7\left[4^{5} 31^{2}\right]+$ $2\left[4^{4} 3^{3}\right]+6\left[4^{5} 32\right]+4\left[4^{6} 1\right]$

$\{13,11,6\} \stackrel{\circ}{=}\left[4^{4} 32^{2} 1^{2}\right]+\left[4^{5} 21^{3}\right]+\left[4^{4} 32^{3}\right]+3\left[4^{4} 3^{2} 21\right]+3\left[4^{5} 2^{2} 1\right]+4\left[4^{5} 31^{2}\right]+$ $2\left[4^{4} 3^{3}\right]+5\left[4^{5} 32\right]+2\left[4^{6} 1\right]$

$\left\{14,8^{2}\right\} \stackrel{\circ}{=}\left[4^{5} 1^{5}\right]+\left[4^{4} 3^{2} 1^{3}\right]+\left[4^{5} 21^{3}\right]+\left[4^{4} 32^{3}\right]+\left[4^{4} 3^{2} 21\right]+3\left[4^{5} 2^{2} 1\right]+$ $2\left[4^{5} 31^{2}\right]+3\left[4^{5} 32\right]+3\left[4^{6} 1\right]$

$\left\{12^{2}, 6\right\} \stackrel{\circ}{=}\left[4^{4} 3^{2} 1^{3}\right]+\left[4^{4} 32^{3}\right]+\left[4^{4} 3^{2} 21\right]+2\left[4^{5} 2^{2} 1\right]+\left[4^{5} 31^{2}\right]+2\left[4^{5} 32\right]+2\left[4^{6} 1\right]$

$\{13,10,7\} \stackrel{\circ}{=} 2\left[4^{4} 32^{2} 1^{2}\right]+2\left[4^{4} 3^{2} 1^{3}\right]+3\left[4^{5} 21^{3}\right]+2\left[4^{4} 32^{3}\right]+4\left[4^{4} 3^{2} 21\right]+$

$6\left[4^{5} 2^{2} 1\right]+7\left[4^{5} 31^{2}\right]+2\left[4^{4} 3^{3}\right]+7\left[4^{5} 32\right]+5\left[4^{6} 1\right]$

$\{13,9,8\} \stackrel{\circ}{=}\left[4^{4} 321^{4}\right]+2\left[4^{4} 32^{2} 1^{2}\right]+\left[4^{4} 3^{2} 1^{3}\right]+3\left[4^{5} 21^{3}\right]+\left[4^{4} 32^{3}\right]+4\left[4^{4} 3^{2} 21\right]+$ $5\left[4^{5} 2^{2} 1\right]+6\left[4^{5} 31^{2}\right]+\left[4^{4} 3^{3}\right]+6\left[4^{5} 32\right]+3\left[4^{6} 1\right]$

$\{12,11,7\} \stackrel{\circ}{=}\left[4^{4} 2^{4} 1\right]+2\left[4^{4} 32^{2} 1^{2}\right]+\left[4^{4} 3^{2} 1^{3}\right]+2\left[4^{5} 21^{3}\right]+\left[4^{4} 32^{3}\right]+4\left[4^{4} 3^{2} 21\right]+$ $4\left[4^{5} 2^{2} 1\right]+5\left[4^{5} 31^{2}\right]+\left[4^{4} 3^{3}\right]+5\left[4^{5} 32\right]+2\left[4^{6} 1\right]$

$\{12,10,8\} \stackrel{\circ}{=}\left[4^{4} 321^{4}\right]+\left[4^{5} 1^{5}\right]+\left[4^{4} 2^{4} 1\right]+2\left[4^{4} 32^{2} 1^{2}\right]+3\left[4^{4} 3^{2} 1^{3}\right]+3\left[4^{5} 21^{3}\right]+$

$3\left[4^{4} 32^{3}\right]+4\left[4^{4} 3^{2} 21\right]+7\left[4^{5} 2^{2} 1\right]+5\left[4^{5} 31^{2}\right]+6\left[4^{5} 32\right]+4\left[4^{6} 1\right]$

$\left\{12,9^{2}\right\} \stackrel{\circ}{=}\left[4^{4} 2^{3} 1^{3}\right]+2\left[4^{4} 32^{2} 1^{2}\right]+\left[4^{4} 3^{2} 1^{3}\right]+2\left[4^{5} 21^{3}\right]+2\left[4^{4} 3^{2} 21\right]+2\left[4^{5} 2^{2} 1\right]+$ $4\left[4^{5} 31^{2}\right]+2\left[4^{4} 3^{3}\right]+2\left[4^{5} 32\right]+\left[4^{6} 1\right]$ 
$\left\{11^{2}, 8\right\} \stackrel{\circ}{=}\left[4^{4} 2^{3} 1^{3}\right]+2\left[4^{4} 32^{2} 1^{2}\right]+2\left[4^{5} 21^{3}\right]+\left[4^{4} 32^{3}\right]+2\left[4^{4} 3^{2} 21\right]+2\left[4^{5} 2^{2} 1\right]+$ $3\left[4^{5} 31^{2}\right]+2\left[4^{4} 3^{3}\right]+2\left[4^{5} 32\right]+\left[4^{6} 1\right]$

$\{11,10,9\} \stackrel{\circ}{=}\left[4^{4} 321^{4}\right]+\left[4^{4} 2^{4} 1\right]+2\left[4^{4} 32^{2} 1^{2}\right]+\left[4^{4} 3^{2} 1^{3}\right]+2\left[4^{5} 21^{3}\right]+\left[4^{4} 32^{3}\right]+$ $2\left[4^{4} 3^{2} 21\right]+3\left[4^{5} 2^{2} 1\right]+3\left[4^{5} 31^{2}\right]+3\left[4^{5} 32\right]+\left[4^{6} 1\right]$

$\left\{10^{3}\right\} \stackrel{\circ}{=}\left[4^{5} 1^{5}\right]+\left[4^{4} 3^{2} 1^{3}\right]+\left[4^{4} 32^{3}\right]+\left[4^{5} 2^{2} 1\right]+\left[4^{6} 1\right]$

$\mathrm{A}=26$

$\{18,8,6\} \stackrel{\circ}{\circ}\left[4^{6} 2\right]$

$\left\{18,7^{2}\right\} \stackrel{\circ}{=}\left[4^{5} 3^{2}\right]$

$\{16,12,4\} \stackrel{\circ}{=}\left[4^{6} 2\right]$

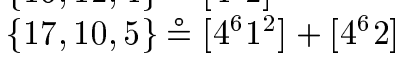

$\{15,13,4\} \stackrel{\circ}{=}\left[4^{5} 3^{2}\right]$

$\{17,9,6\} \stackrel{\circ}{=}\left[4^{5} 321\right]+\left[4^{6} 1^{2}\right]+\left[4^{5} 3^{2}\right]+\left[4^{6} 2\right]$

$\left\{14^{2}, 4\right\} \stackrel{\circ}{=}\left[4^{6} 2\right]$

$\{16,11,5\} \stackrel{\circ}{=}\left[4^{5} 321\right]+\left[4^{6} 1^{2}\right]+\left[4^{5} 3^{2}\right]+\left[4^{6} 2\right]$

$\{17,8,7\} \stackrel{\circ}{=}\left[4^{5} 321\right]+\left[4^{6} 1^{2}\right]+\left[4^{5} 3^{2}\right]+2\left[4^{6} 2\right]$

$\{15,12,5\} \stackrel{\circ}{=} 2\left[4^{5} 321\right]+\left[4^{6} 1^{2}\right]+\left[4^{5} 3^{2}\right]+2\left[4^{6} 2\right]$

$\{16,10,6\} \stackrel{\circ}{=}\left[4^{5} 31^{3}\right]+\left[4^{5} 2^{3}\right]+3\left[4^{5} 321\right]+2\left[4^{6} 1^{2}\right]+\left[4^{5} 3^{2}\right]+4\left[4^{6} 2\right]$

$\{14,13,5\} \stackrel{\circ}{=}\left[4^{4} 3^{3} 1\right]+\left[4^{5} 321\right]+\left[4^{6} 1^{2}\right]+\left[4^{5} 3^{2}\right]+\left[4^{6} 2\right]$

$\{16,9,7\} \stackrel{\circ}{=}\left[4^{5} 2^{2} 1^{2}\right]+\left[4^{5} 31^{3}\right]+\left[4^{4} 3^{3} 1\right]+4\left[4^{5} 321\right]+3\left[4^{6} 1^{2}\right]+3\left[4^{5} 3^{2}\right]+3\left[4^{6} 2\right]$

$\{15,11,6\} \stackrel{\circ}{=}\left[4^{5} 2^{2} 1^{2}\right]+\left[4^{5} 31^{3}\right]+\left[4^{4} 3^{2} 2^{2}\right]+\left[4^{5} 2^{3}\right]+\left[4^{4} 3^{3} 1\right]+5\left[4^{5} 321\right]+$

$3\left[4^{6} 1^{2}\right]+3\left[4^{5} 3^{2}\right]+3\left[4^{6} 2\right]$

$\left\{16,8^{2}\right\} \stackrel{\circ}{=}\left[4^{5} 31^{3}\right]+\left[4^{5} 2^{3}\right]+2\left[4^{5} 321\right]+\left[4^{6} 1^{2}\right]+3\left[4^{6} 2\right]$

$\{14,12,6\} \stackrel{\circ}{=}\left[4^{4} 3^{2} 21^{2}\right]+\left[4^{5} 31^{3}\right]+\left[4^{4} 3^{2} 2^{2}\right]+2\left[4^{5} 2^{3}\right]+\left[4^{4} 3^{3} 1\right]+5\left[4^{5} 321\right]+$ $2\left[4^{6} 1^{2}\right]+2\left[4^{5} 3^{2}\right]+5\left[4^{6} 2\right]$

$\{15,10,7\} \stackrel{\circ}{=}\left[4^{4} 3^{2} 21^{2}\right]+2\left[4^{5} 2^{2} 1^{2}\right]+3\left[4^{5} 31^{3}\right]+\left[4^{4} 3^{2} 2^{2}\right]+2\left[4^{5} 2^{3}\right]+\left[4^{4} 3^{3} 1\right]+$

$8\left[4^{5} 321\right]+5\left[4^{6} 1^{2}\right]+4\left[4^{5} 3^{2}\right]+6\left[4^{6} 2\right]$

$\left\{13^{2}, 6\right\} \stackrel{\circ}{=}\left[4^{5} 2^{2} 1^{2}\right]+\left[4^{4} 3^{2} 2^{2}\right]+\left[4^{4} 3^{3} 1\right]+2\left[4^{5} 321\right]+\left[4^{6} 1^{2}\right]+2\left[4^{5} 3^{2}\right]$

$\{15,9,8\} \stackrel{\circ}{=}\left[4^{5} 21^{4}\right]+\left[4^{4} 3^{2} 21^{2}\right]+2\left[4^{5} 2^{2} 1^{2}\right]+2\left[4^{5} 31^{3}\right]+\left[4^{4} 3^{2} 2^{2}\right]+\left[4^{5} 2^{3}\right]+$

$\left[4^{4} 3^{3} 1\right]+7\left[4^{5} 321\right]+4\left[4^{6} 1^{2}\right]+3\left[4^{5} 3^{2}\right]+4\left[4^{6} 2\right]$

$\{14,11,7\} \stackrel{\circ}{=}\left[4^{4} 32^{3} 1\right]+2\left[4^{4} 3^{2} 21^{2}\right]+3\left[4^{5} 2^{2} 1^{2}\right]+3\left[4^{5} 31^{3}\right]+2\left[4^{4} 3^{2} 2^{2}\right]+$

$2\left[4^{5} 2^{3}\right]+3\left[4^{4} 3^{3} 1\right]+10\left[4^{5} 321\right]+5\left[4^{6} 1^{2}\right]+5\left[4^{5} 3^{2}\right]+5\left[4^{6} 2\right]$

$\{13,12,7\} \stackrel{\circ}{=}\left[4^{4} 32^{3} 1\right]+2\left[4^{4} 3^{2} 21^{2}\right]+2\left[4^{5} 2^{2} 1^{2}\right]+2\left[4^{5} 31^{3}\right]+2\left[4^{4} 3^{2} 2^{2}\right]+$

$2\left[4^{5} 2^{3}\right]+2\left[4^{4} 3^{3} 1\right]+7\left[4^{5} 321\right]+3\left[4^{6} 1^{2}\right]+3\left[4^{5} 3^{2}\right]+4\left[4^{6} 2\right]$

$\{14,10,8\} \stackrel{\circ}{=}\left[4^{4} 3^{2} 1^{4}\right]+\left[4^{5} 21^{4}\right]+\left[4^{4} 32^{3} 1\right]+3\left[4^{4} 3^{2} 21^{2}\right]+3\left[4^{5} 2^{2} 1^{2}\right]+5\left[4^{5} 31^{3}\right]+$ $2\left[4^{4} 3^{2} 2^{2}\right]+5\left[4^{5} 2^{3}\right]+2\left[4^{4} 3^{3} 1\right]+11\left[4^{5} 321\right]+5\left[4^{6} 1^{2}\right]+3\left[4^{5} 3^{2}\right]+8\left[4^{6} 2\right]$

$\left\{14,9^{2}\right\} \stackrel{\circ}{=}\left[4^{4} 32^{2} 1^{3}\right]+\left[4^{4} 3^{2} 21^{2}\right]+3\left[4^{5} 2^{2} 1^{2}\right]+2\left[4^{5} 31^{3}\right]+\left[4^{4} 3^{2} 2^{2}\right]+2\left[4^{4} 3^{3} 1\right]+$ $5\left[4^{5} 321\right]+3\left[4^{6} 1^{2}\right]+3\left[4^{5} 3^{2}\right]+\left[4^{6} 2\right]$

$\{13,11,8\} \stackrel{\circ}{=}\left[4^{4} 32^{2} 1^{3}\right]+\left[4^{5} 21^{4}\right]+2\left[4^{4} 32^{3} 1\right]+3\left[4^{4} 3^{2} 21^{2}\right]+5\left[4^{5} 2^{2} 1^{2}\right]+$ $4\left[4^{5} 31^{3}\right]+4\left[4^{4} 3^{2} 2^{2}\right]+3\left[4^{5} 2^{3}\right]+3\left[4^{4} 3^{3} 1\right]+11\left[4^{5} 321\right]+5\left[4^{6} 1^{2}\right]+5\left[4^{5} 3^{2}\right]+$ $4\left[4^{6} 2\right]$

$\left\{12^{2}, 8\right\} \stackrel{\circ}{=}\left[4^{4} 3^{2} 1^{4}\right]+\left[4^{4} 2^{5}\right]+\left[4^{4} 32^{3} 1\right]+2\left[4^{4} 3^{2} 21^{2}\right]+\left[4^{5} 2^{2} 1^{2}\right]+2\left[4^{5} 31^{3}\right]+$

$\left[4^{4} 3^{2} 2^{2}\right]+3\left[4^{5} 2^{3}\right]+\left[4^{4} 3^{3} 1\right]+5\left[4^{5} 321\right]+\left[4^{6} 1^{2}\right]+4\left[4^{6} 2\right]$

$\{13,10,9\} \stackrel{\circ}{=}\left[4^{4} 32^{2} 1^{3}\right]+\left[4^{4} 3^{2} 1^{4}\right]+\left[4^{5} 21^{4}\right]+2\left[4^{4} 32^{3} 1\right]+3\left[4^{4} 3^{2} 21^{2}\right]+$

$4\left[4^{5} 2^{2} 1^{2}\right]+4\left[4^{5} 31^{3}\right]+2\left[4^{4} 3^{2} 2^{2}\right]+3\left[4^{5} 2^{3}\right]+2\left[4^{4} 3^{3} 1\right]+9\left[4^{5} 321\right]+4\left[4^{6} 1^{2}\right]+$ $3\left[4^{5} 3^{2}\right]+4\left[4^{6} 2\right]$

$\{12,11,9\} \stackrel{\circ}{=}\left[4^{4} 2^{4} 1^{2}\right]+\left[4^{4} 32^{2} 1^{3}\right]+\left[4^{5} 21^{4}\right]+2\left[4^{4} 32^{3} 1\right]+3\left[4^{4} 3^{2} 21^{2}\right]+$ $4\left[4^{5} 2^{2} 1^{2}\right]+3\left[4^{5} 31^{3}\right]+2\left[4^{4} 3^{2} 2^{2}\right]+2\left[4^{5} 2^{3}\right]+2\left[4^{4} 3^{3} 1\right]+7\left[4^{5} 321\right]+3\left[4^{6} 1^{2}\right]+$ $3\left[4^{5} 3^{2}\right]+2\left[4^{6} 2\right]$

$\left\{12,10^{2}\right\} \stackrel{\circ}{=}\left[4^{4} 3^{2} 1^{4}\right]+\left[4^{5} 21^{4}\right]+\left[4^{4} 2^{5}\right]+\left[4^{4} 32^{3} 1\right]+2\left[4^{4} 3^{2} 21^{2}\right]+\left[4^{5} 2^{2} 1^{2}\right]+$ $2\left[4^{5} 31^{3}\right]+\left[4^{4} 3^{2} 2^{2}\right]+3\left[4^{5} 2^{3}\right]+3\left[4^{5} 321\right]+\left[4^{6} 1^{2}\right]+3\left[4^{6} 2\right]$

$\left\{11^{2}, 10\right\} \stackrel{\circ}{=}\left[4^{4} 32^{2} 1^{3}\right]+\left[4^{4} 32^{3} 1\right]+2\left[4^{5} 2^{2} 1^{2}\right]+\left[4^{5} 31^{3}\right]+\left[4^{4} 3^{2} 2^{2}\right]+\left[4^{4} 3^{3} 1\right]+$ $2\left[4^{5} 321\right]+\left[4^{6} 1^{2}\right]+\left[4^{5} 3^{2}\right]$

$\mathrm{A}=27$

$\{19,8,7\} \stackrel{\circ}{=}\left[4^{6} 3\right]$

$\{16,14,4\} \stackrel{\circ}{=}\left[4^{6} 3\right]$ 
$\{18,10,6\} \stackrel{\circ}{=}\left[4^{6} 21\right]+\left[4^{6} 3\right]$

$\{17,12,5\} \stackrel{\circ}{=}\left[4^{6} 21\right]+\left[4^{6} 3\right]$

$\{18,9,7\} \stackrel{\circ}{=}\left[4^{5} 3^{2} 1\right]+\left[4^{6} 21\right]+\left[4^{6} 3\right]$

$\{16,13,5\} \stackrel{\circ}{=}\left[4^{5} 3^{2} 1\right]+\left[4^{6} 21\right]+\left[4^{6} 3\right]$

$\left\{18,8^{2}\right\} \stackrel{\circ}{=}\left[4^{6} 21\right]+\left[4^{6} 3\right]$

$\{17,11,6\} \stackrel{\circ}{=}\left[4^{6} 1^{3}\right]+\left[4^{5} 32^{2}\right]+\left[4^{5} 3^{2} 1\right]+2\left[4^{6} 21\right]+\left[4^{6} 3\right]$

$\{15,14,5\} \stackrel{\circ}{=}\left[4^{5} 3^{2} 1\right]+\left[4^{6} 21\right]+\left[4^{6} 3\right]$

$\{17,10,7\} \stackrel{\circ}{=}\left[4^{5} 321^{2}\right]+\left[4^{6} 1^{3}\right]+\left[4^{5} 32^{2}\right]+2\left[4^{5} 3^{2} 1\right]+4\left[4^{6} 21\right]+3\left[4^{6} 3\right]$

$\{16,12,6\} \stackrel{\circ}{=}\left[4^{5} 321^{2}\right]+2\left[4^{5} 32^{2}\right]+2\left[4^{5} 3^{2} 1\right]+4\left[4^{6} 21\right]+3\left[4^{6} 3\right]$

$\{17,9,8\} \stackrel{\circ}{=}\left[4^{5} 321^{2}\right]+\left[4^{6} 1^{3}\right]+\left[4^{5} 32^{2}\right]+2\left[4^{5} 3^{2} 1\right]+3\left[4^{6} 21\right]+2\left[4^{6} 3\right]$

$\{15,13,6\} \stackrel{\circ}{\circ}\left[4^{5} 321^{2}\right]+\left[4^{6} 1^{3}\right]+\left[4^{4} 3^{3} 2\right]+2\left[4^{5} 32^{2}\right]+3\left[4^{5} 3^{2} 1\right]+3\left[4^{6} 21\right]+2\left[4^{6} 3\right]$

$\{16,11,7\} \stackrel{\circ}{=}\left[4^{5} 2^{3} 1\right]+3\left[4^{5} 321^{2}\right]+2\left[4^{6} 1^{3}\right]+\left[4^{4} 3^{3} 2\right]+3\left[4^{5} 32^{2}\right]+5\left[4^{5} 3^{2} 1\right]+$

$6\left[4^{6} 21\right]+4\left[4^{6} 3\right]$

$\left\{14^{2}, 6\right\} \stackrel{\circ}{=}\left[4^{4} 3^{3} 1^{2}\right]+\left[4^{5} 32^{2}\right]+\left[4^{5} 3^{2} 1\right]+2\left[4^{6} 21\right]+2\left[4^{6} 3\right]$

$\{16,10,8\} \stackrel{\circ}{=}\left[4^{5} 31^{4}\right]+\left[4^{5} 2^{3} 1\right]+\left[4^{4} 3^{3} 1^{2}\right]+4\left[4^{5} 321^{2}\right]+2\left[4^{6} 1^{3}\right]+4\left[4^{5} 32^{2}\right]+$ $4\left[4^{5} 3^{2} 1\right]+8\left[4^{6} 21\right]+5\left[4^{6} 3\right]$

$\{15,12,7\} \stackrel{\circ}{=}\left[4^{4} 3^{2} 2^{2} 1\right]+\left[4^{5} 2^{3} 1\right]+\left[4^{4} 3^{3} 1^{2}\right]+4\left[4^{5} 321^{2}\right]+2\left[4^{6} 1^{3}\right]+\left[4^{4} 3^{3} 2\right]+$ $5\left[4^{5} 32^{2}\right]+6\left[4^{5} 3^{2} 1\right]+8\left[4^{6} 21\right]+5\left[4^{6} 3\right]$

$\left\{16,9^{2}\right\} \stackrel{\circ}{=}\left[4^{5} 2^{2} 1^{3}\right]+2\left[4^{5} 321^{2}\right]+\left[4^{6} 1^{3}\right]+\left[4^{4} 3^{3} 2\right]+\left[4^{5} 32^{2}\right]+3\left[4^{5} 3^{2} 1\right]+$

$3\left[4^{6} 21\right]+\left[4^{6} 3\right]$

$\{14,13,7\} \stackrel{\circ}{=}\left[4^{4} 3^{2} 2^{2} 1\right]+\left[4^{5} 2^{3} 1\right]+\left[4^{4} 3^{3} 1^{2}\right]+3\left[4^{5} 321^{2}\right]+\left[4^{6} 1^{3}\right]+2\left[4^{4} 3^{3} 2\right]+$ $3\left[4^{5} 32^{2}\right]+5\left[4^{5} 3^{2} 1\right]+5\left[4^{6} 21\right]+3\left[4^{6} 3\right]$

$\{15,11,8\} \stackrel{\circ}{=}\left[4^{5} 2^{2} 1^{3}\right]+\left[4^{5} 31^{4}\right]+2\left[4^{4} 3^{2} 2^{2} 1\right]+2\left[4^{5} 2^{3} 1\right]+\left[4^{4} 3^{3} 1^{2}\right]+7\left[4^{5} 321^{2}\right]+$ $4\left[4^{6} 1^{3}\right]+2\left[4^{4} 3^{3} 2\right]+7\left[4^{5} 32^{2}\right]+8\left[4^{5} 3^{2} 1\right]+10\left[4^{6} 21\right]+5\left[4^{6} 3\right]$

$\{15,10,9\} \stackrel{\circ}{=}\left[4^{4} 3^{2} 21^{3}\right]+\left[4^{5} 2^{2} 1^{3}\right]+\left[4^{5} 31^{4}\right]+\left[4^{4} 3^{2} 2^{2} 1\right]+2\left[4^{5} 2^{3} 1\right]+\left[4^{4} 3^{3} 1^{2}\right]+$

$6\left[4^{5} 321^{2}\right]+3\left[4^{6} 1^{3}\right]+\left[4^{4} 3^{3} 2\right]+5\left[4^{5} 32^{2}\right]+6\left[4^{5} 3^{2} 1\right]+8\left[4^{6} 21\right]+4\left[4^{6} 3\right]$

$\{14,12,8\} \stackrel{\circ}{=}\left[4^{4} 3^{2} 21^{3}\right]+\left[4^{5} 31^{4}\right]+\left[4^{4} 32^{4}\right]+2\left[4^{4} 3^{2} 2^{2} 1\right]+3\left[4^{5} 2^{3} 1\right]+3\left[4^{4} 3^{3} 1^{2}\right]+$

$7\left[4^{5} 321^{2}\right]+2\left[4^{6} 1^{3}\right]+2\left[4^{4} 3^{3} 2\right]+8\left[4^{5} 32^{2}\right]+7\left[4^{5} 3^{2} 1\right]+10\left[4^{6} 21\right]+6\left[4^{6} 3\right]$

$\left\{13^{2}, 8\right\} \stackrel{\circ}{=}\left[4^{5} 2^{2} 1^{3}\right]+2\left[4^{4} 3^{2} 2^{2} 1\right]+\left[4^{5} 2^{3} 1\right]+3\left[4^{5} 321^{2}\right]+2\left[4^{6} 1^{3}\right]+2\left[4^{4} 3^{3} 2\right]+$

$3\left[4^{5} 32^{2}\right]+4\left[4^{5} 3^{2} 1\right]+3\left[4^{6} 21\right]+\left[4^{6} 3\right]$

$\{14,11,9\} \stackrel{\circ}{=}\left[4^{4} 32^{3} 1^{2}\right]+\left[4^{4} 3^{2} 21^{3}\right]+2\left[4^{5} 2^{2} 1^{3}\right]+\left[4^{5} 31^{4}\right]+3\left[4^{4} 3^{2} 2^{2} 1\right]+$

$4\left[4^{5} 2^{3} 1\right]+2\left[4^{4} 3^{3} 1^{2}\right]+9\left[4^{5} 321^{2}\right]+4\left[4^{6} 1^{3}\right]+3\left[4^{4} 3^{3} 2\right]+7\left[4^{5} 32^{2}\right]+9\left[4^{5} 3^{2} 1\right]+$ $9\left[4^{6} 21\right]+4\left[4^{6} 3\right]$

$\left\{14,10^{2}\right\} \stackrel{\circ}{=}\left[4^{4} 3^{2} 21^{3}\right]+2\left[4^{5} 31^{4}\right]+\left[4^{4} 32^{4}\right]+\left[4^{4} 3^{2} 2^{2} 1\right]+2\left[4^{5} 2^{3} 1\right]+2\left[4^{4} 3^{3} 1^{2}\right]+$ $4\left[4^{5} 321^{2}\right]+\left[4^{6} 1^{3}\right]+4\left[4^{5} 32^{2}\right]+2\left[4^{5} 3^{2} 1\right]+5\left[4^{6} 21\right]+3\left[4^{6} 3\right]$

$\{13,12,9\} \stackrel{\circ}{=}\left[4^{4} 32^{3} 1^{2}\right]+\left[4^{4} 3^{2} 21^{3}\right]+\left[4^{5} 2^{2} 1^{3}\right]+\left[4^{5} 31^{4}\right]+\left[4^{4} 32^{4}\right]+3\left[4^{4} 3^{2} 2^{2} 1\right]+$

$3\left[4^{5} 2^{3} 1\right]+2\left[4^{4} 3^{3} 1^{2}\right]+7\left[4^{5} 321^{2}\right]+2\left[4^{6} 1^{3}\right]+2\left[4^{4} 3^{3} 2\right]+6\left[4^{5} 32^{2}\right]+6\left[4^{5} 3^{2} 1\right]+$

$7\left[4^{6} 21\right]+3\left[4^{6} 3\right]$

$\{13,11,10\} \stackrel{\circ}{=}\left[4^{4} 32^{3} 1^{2}\right]+\left[4^{4} 3^{2} 21^{3}\right]+2\left[4^{5} 2^{2} 1^{3}\right]+\left[4^{5} 31^{4}\right]+\left[4^{4} 32^{4}\right]+3\left[4^{4} 3^{2} 2^{2} 1\right]+$ $3\left[4^{5} 2^{3} 1\right]+\left[4^{4} 3^{3} 1^{2}\right]+6\left[4^{5} 321^{2}\right]+3\left[4^{6} 1^{3}\right]+2\left[4^{4} 3^{3} 2\right]+5\left[4^{5} 32^{2}\right]+5\left[4^{5} 3^{2} 1\right]+$ $5\left[4^{6} 21\right]+2\left[4^{6} 3\right]$

$\left\{12^{2}, 10\right\} \stackrel{\circ}{=}\left[4^{4} 2^{5} 1\right]+\left[4^{4} 3^{2} 21^{3}\right]+\left[4^{5} 31^{4}\right]+\left[4^{4} 32^{4}\right]+\left[4^{4} 3^{2} 2^{2} 1\right]+2\left[4^{5} 2^{3} 1\right]+$

$2\left[4^{4} 3^{3} 1^{2}\right]+3\left[4^{5} 321^{2}\right]+3\left[4^{5} 32^{2}\right]+\left[4^{5} 3^{2} 1\right]+3\left[4^{6} 21\right]+2\left[4^{6} 3\right]$

$\left\{12,11^{2}\right\} \stackrel{\circ}{\doteq}\left[4^{4} 32^{3} 1^{2}\right]+\left[4^{5} 2^{2} 1^{3}\right]+\left[4^{4} 3^{2} 2^{2} 1\right]+\left[4^{5} 2^{3} 1\right]+2\left[4^{5} 321^{2}\right]+\left[4^{6} 1^{3}\right]+$

$\left[4^{4} 3^{3} 2\right]+\left[4^{5} 32^{2}\right]+2\left[4^{5} 3^{2} 1\right]+\left[4^{6} 21\right]$

$\mathrm{A}=28$

$\left\{20,8^{2}\right\} \stackrel{\circ}{=}\left[4^{7}\right]$

$\left\{16^{2}, 4\right\} \stackrel{\circ}{=}\left[4^{7}\right]$

$\{19,10,7\} \stackrel{\circ}{=}\left[4^{6} 31\right]+\left[4^{7}\right]$

$\{17,14,5\} \stackrel{\circ}{=}\left[4^{6} 31\right]+\left[4^{7}\right]$

$\{18,12,6\} \stackrel{\circ}{\circ}\left[4^{6} 2^{2}\right]+\left[4^{6} 31\right]+\left[4^{7}\right]$

$\{19,9,8\} \stackrel{\circ}{=}\left[4^{6} 31\right]$

$\{16,15,5\} \stackrel{\circ}{=}\left[4^{6} 31\right]$

$\{18,11,7\} \stackrel{\circ}{\doteq}\left[4^{6} 21^{2}\right]+\left[4^{5} 3^{2} 2\right]+\left[4^{6} 2^{2}\right]+2\left[4^{6} 31\right]$

$\{17,13,6\} \stackrel{\circ}{=}\left[4^{6} 21^{2}\right]+\left[4^{5} 3^{2} 2\right]+\left[4^{6} 2^{2}\right]+2\left[4^{6} 31\right]$

$\{18,10,8\} \stackrel{\circ}{\circ}\left[4^{5} 3^{2} 1^{2}\right]+\left[4^{6} 21^{2}\right]+2\left[4^{6} 2^{2}\right]+3\left[4^{6} 31\right]+2\left[4^{7}\right]$ 
$\{16,14,6\} \stackrel{\circ}{=}\left[4^{5} 3^{2} 1^{2}\right]+\left[4^{5} 3^{2} 2\right]+2\left[4^{6} 2^{2}\right]+3\left[4^{6} 31\right]+2\left[4^{7}\right]$

$\left\{18,9^{2}\right\} \stackrel{\circ}{=}\left[4^{6} 21^{2}\right]+\left[4^{5} 3^{2} 2\right]+\left[4^{6} 31\right]$

$\left\{15^{2}, 6\right\} \stackrel{\circ}{=}\left[4^{6} 21^{2}\right]+\left[4^{5} 3^{2} 2\right]+\left[4^{6} 31\right]$

$\{17,12,7\} \stackrel{\circ}{=}\left[4^{5} 32^{2} 1\right]+\left[4^{5} 3^{2} 1^{2}\right]+2\left[4^{6} 21^{2}\right]+2\left[4^{5} 3^{2} 2\right]+3\left[4^{6} 2^{2}\right]+5\left[4^{6} 31\right]+2\left[4^{7}\right]$

$\{17,11,8\} \stackrel{\circ}{=}\left[4^{6} 1^{4}\right]+2\left[4^{5} 32^{2} 1\right]+2\left[4^{5} 3^{2} 1^{2}\right]+4\left[4^{6} 21^{2}\right]+3\left[4^{5} 3^{2} 2\right]+3\left[4^{6} 2^{2}\right]+$

$6\left[4^{6} 31\right]+2\left[4^{7}\right]$

$\{16,13,7\} \stackrel{\circ}{=} 2\left[4^{5} 32^{2} 1\right]+2\left[4^{5} 3^{2} 1^{2}\right]+3\left[4^{6} 21^{2}\right]+\left[4^{4} 3^{4}\right]+4\left[4^{5} 3^{2} 2\right]+3\left[4^{6} 2^{2}\right]+$

$6\left[4^{6} 31\right]+2\left[4^{7}\right]$

$\{17,10,9\} \stackrel{\circ}{=}\left[4^{5} 321^{3}\right]+\left[4^{5} 32^{2} 1\right]+2\left[4^{5} 3^{2} 1^{2}\right]+3\left[4^{6} 21^{2}\right]+2\left[4^{5} 3^{2} 2\right]+3\left[4^{6} 2^{2}\right]+$

$5\left[4^{6} 31\right]+\left[4^{7}\right]$

$\{15,14,7\} \stackrel{\circ}{=}\left[4^{4} 3^{3} 21\right]+\left[4^{5} 32^{2} 1\right]+2\left[4^{5} 3^{2} 1^{2}\right]+2\left[4^{6} 21^{2}\right]+3\left[4^{5} 3^{2} 2\right]+3\left[4^{6} 2^{2}\right]+$ $5\left[4^{6} 31\right]+\left[4^{7}\right]$

$\{16,12,8\} \stackrel{\circ}{=}\left[4^{5} 321^{3}\right]+\left[4^{5} 2^{4}\right]+\left[4^{4} 3^{3} 21\right]+4\left[4^{5} 32^{2} 1\right]+5\left[4^{5} 3^{2} 1^{2}\right]+5\left[4^{6} 21^{2}\right]+$ $5\left[4^{5} 3^{2} 2\right]+8\left[4^{6} 2^{2}\right]+9\left[4^{6} 31\right]+4\left[4^{7}\right]$

$\{16,11,9\} \stackrel{\circ}{=}\left[4^{5} 2^{3} 1^{2}\right]+2\left[4^{5} 321^{3}\right]+\left[4^{6} 1^{4}\right]+\left[4^{4} 3^{3} 21\right]+5\left[4^{5} 32^{2} 1\right]+4\left[4^{5} 3^{2} 1^{2}\right]+$

$7\left[4^{6} 21^{2}\right]+\left[4^{4} 3^{4}\right]+6\left[4^{5} 3^{2} 2\right]+5\left[4^{6} 2^{2}\right]+9\left[4^{6} 31\right]+\left[4^{7}\right]$

$\{15,13,8\} \stackrel{\circ}{=}\left[4^{5} 321^{3}\right]+\left[4^{6} 1^{4}\right]+\left[4^{4} 3^{2} 2^{3}\right]+2\left[4^{4} 3^{3} 21\right]+5\left[4^{5} 32^{2} 1\right]+4\left[4^{5} 3^{2} 1^{2}\right]+$

$6\left[4^{6} 21^{2}\right]+\left[4^{4} 3^{4}\right]+7\left[4^{5} 3^{2} 2\right]+5\left[4^{6} 2^{2}\right]+9\left[4^{6} 31\right]+\left[4^{7}\right]$

$\left\{16,10^{2}\right\} \stackrel{\circ}{=}\left[4^{4} 3^{3} 1^{3}\right]+\left[4^{5} 321^{3}\right]+\left[4^{6} 1^{4}\right]+\left[4^{5} 2^{4}\right]+2\left[4^{5} 32^{2} 1\right]+3\left[4^{5} 3^{2} 1^{2}\right]+$

$2\left[4^{6} 21^{2}\right]+\left[4^{5} 3^{2} 2\right]+4\left[4^{6} 2^{2}\right]+4\left[4^{6} 31\right]+3\left[4^{7}\right]$

$\left\{14^{2}, 8\right\} \stackrel{\circ}{=}\left[4^{4} 3^{3} 1^{3}\right]+\left[4^{5} 2^{4}\right]+\left[4^{4} 3^{3} 21\right]+2\left[4^{5} 32^{2} 1\right]+3\left[4^{5} 3^{2} 1^{2}\right]+\left[4^{6} 21^{2}\right]+$

$\left[4^{4} 3^{4}\right]+2\left[4^{5} 3^{2} 2\right]+4\left[4^{6} 2^{2}\right]+4\left[4^{6} 31\right]+3\left[4^{7}\right]$

$\{15,12,9\} \stackrel{\circ}{=}\left[4^{4} 3^{2} 2^{2} 1^{2}\right]+\left[4^{5} 2^{3} 1^{2}\right]+\left[4^{4} 3^{3} 1^{3}\right]+2\left[4^{5} 321^{3}\right]+\left[4^{6} 1^{4}\right]+\left[4^{4} 3^{2} 2^{3}\right]+$

$\left[4^{5} 2^{4}\right]+2\left[4^{4} 3^{3} 21\right]+8\left[4^{5} 32^{2} 1\right]+7\left[4^{5} 3^{2} 1^{2}\right]+8\left[4^{6} 21^{2}\right]+\left[4^{4} 3^{4}\right]+8\left[4^{5} 3^{2} 2\right]+$

$8\left[4^{6} 2^{2}\right]+11\left[4^{6} 31\right]+3\left[4^{7}\right]$

$\{15,11,10\} \stackrel{\circ}{=}\left[4^{4} 3^{2} 2^{2} 1^{2}\right]+\left[4^{5} 2^{3} 1^{2}\right]+3\left[4^{5} 321^{3}\right]+\left[4^{6} 1^{4}\right]+\left[4^{4} 3^{2} 2^{3}\right]+\left[4^{5} 2^{4}\right]+$

$2\left[4^{4} 3^{3} 21\right]+6\left[4^{5} 32^{2} 1\right]+5\left[4^{5} 3^{2} 1^{2}\right]+7\left[4^{6} 21^{2}\right]+6\left[4^{5} 3^{2} 2\right]+5\left[4^{6} 2^{2}\right]+7\left[4^{6} 31\right]+\left[4^{7}\right]$

$\{14,13,9\} \stackrel{\circ}{=}\left[4^{4} 3^{2} 2^{2} 1^{2}\right]+\left[4^{5} 2^{3} 1^{2}\right]+2\left[4^{5} 321^{3}\right]+\left[4^{4} 3^{2} 2^{3}\right]+\left[4^{5} 2^{4}\right]+3\left[4^{4} 3^{3} 21\right]+$

$6\left[4^{5} 32^{2} 1\right]+5\left[4^{5} 3^{2} 1^{2}\right]+6\left[4^{6} 21^{2}\right]+\left[4^{4} 3^{4}\right]+7\left[4^{5} 3^{2} 2\right]+5\left[4^{6} 2^{2}\right]+7\left[4^{6} 31\right]+$

$\left[4^{7}\right]$

$\{14,12,10\} \stackrel{\circ}{=}\left[4^{4} 32^{4} 1\right]+\left[4^{4} 3^{2} 2^{2} 1^{2}\right]+\left[4^{5} 2^{3} 1^{2}\right]+2\left[4^{4} 3^{3} 1^{3}\right]+3\left[4^{5} 321^{3}\right]+$

$\left[4^{6} 1^{4}\right]+\left[4^{4} 3^{2} 2^{3}\right]+3\left[4^{5} 2^{4}\right]+3\left[4^{4} 3^{3} 21\right]+8\left[4^{5} 32^{2} 1\right]+7\left[4^{5} 3^{2} 1^{2}\right]+5\left[4^{6} 21^{2}\right]+$

$\left[4^{4} 3^{4}\right]+5\left[4^{5} 3^{2} 2\right]+8\left[4^{6} 2^{2}\right]+8\left[4^{6} 31\right]+3\left[4^{7}\right]$

$\left\{14,11^{2}\right\} \stackrel{\circ}{=}\left[4^{4} 3^{2} 2^{2} 1^{2}\right]+2\left[4^{5} 2^{3} 1^{2}\right]+\left[4^{5} 321^{3}\right]+\left[4^{6} 1^{4}\right]+\left[4^{4} 3^{2} 2^{3}\right]+\left[4^{4} 3^{3} 21\right]+$

$4\left[4^{5} 32^{2} 1\right]+2\left[4^{5} 3^{2} 1^{2}\right]+4\left[4^{6} 21^{2}\right]+\left[4^{4} 3^{4}\right]+4\left[4^{5} 3^{2} 2\right]+\left[4^{6} 2^{2}\right]+3\left[4^{6} 31\right]$

$\left\{13^{2}, 10\right\} \stackrel{\circ}{=}\left[4^{4} 3^{2} 2^{2} 1^{2}\right]+\left[4^{5} 2^{3} 1^{2}\right]+\left[4^{5} 321^{3}\right]+\left[4^{6} 1^{4}\right]+2\left[4^{4} 3^{2} 2^{3}\right]+\left[4^{4} 3^{3} 21\right]+$

$4\left[4^{5} 32^{2} 1\right]+2\left[4^{5} 3^{2} 1^{2}\right]+4\left[4^{6} 21^{2}\right]+\left[4^{4} 3^{4}\right]+4\left[4^{5} 3^{2} 2\right]+\left[4^{6} 2^{2}\right]+3\left[4^{6} 31\right]$

$\{13,12,11\} \stackrel{\circ}{=}\left[4^{4} 32^{4} 1\right]+\left[4^{4} 3^{2} 2^{2} 1^{2}\right]+\left[4^{5} 2^{3} 1^{2}\right]+2\left[4^{5} 321^{3}\right]+\left[4^{4} 3^{2} 2^{3}\right]+\left[4^{5} 2^{4}\right]+$

$2\left[4^{4} 3^{3} 21\right]+4\left[4^{5} 32^{2} 1\right]+3\left[4^{5} 3^{2} 1^{2}\right]+3\left[4^{6} 21^{2}\right]+3\left[4^{5} 3^{2} 2\right]+3\left[4^{6} 2^{2}\right]+3\left[4^{6} 31\right]$

$\left\{12^{3}\right\} \stackrel{\circ}{=}\left[4^{4} 2^{6}\right]+\left[4^{4} 3^{3} 1^{3}\right]+\left[4^{5} 2^{4}\right]+\left[4^{5} 32^{2} 1\right]+\left[4^{5} 3^{2} 1^{2}\right]+\left[4^{6} 2^{2}\right]+2\left[4^{7}\right]$

\section{References}

1. D.E. Littlewood, The Theory of Group Characters, Oxford Univ. Press, Oxford, 1958.

2. B.G. Wybourne, Symmetry Principles and Atomic Spectroscopy, Wiley, New York, 1970.

3. V. Vanagas, Algebraic Methods in Nuclear Theory (in Russian), Mints, Vilnius(Lituânia), 1971.

4. I. Schur, "Über eine Klasse von Matrizen sich einer gegebenen Matrix zuordnen lassen" , Inaugural-Dissertation, Berlin, 1901.

5. I. Schur, Neue Begrundung der Theorie der Gruppencharaktere, Sitz Ber.Preuss. Akad., Berlin 1905.

6. I. Schur, Über die Darstellung der symmetrid- chen Gruppe durch lineare homogene Substitutionen, Sitz Ber. Preuss. Akad., Berlin 1908.

7. G. Frobenius, Über die Charaktere der Symmetrischen Gruppe, Sitz Ber. Preuss. Akad., Berlin, 1900.

8. D.E. Littlewood, Proc. London Math. Soc. 43, 226 (1937).

9. D.E. Littlewood, Trans. Roy. Soc. (London), A 239, 387 (1943).

10. D.E. Littlewood, Trans. Roy. Soc. (London), A 239, 305 (1943).

11. G.B. Robinson, Can. J. Math. 1, 166 (1949).

12. D.E. Littlewood, J. London Math. Soc. 32, 18 (1957). 
13. D.E. Littlewood, Can. J. Math. 10, 1 (1958).

14. D. E. Littlewood, J. London Math. Soc. 11, 49 (1936).

15. E. M. Ibrahim, Proc. Math. Phys. Soc. Egypt, 5, 85 (1954).

16. E. M. Ibrahim, Proc. Math. Phys. Soc. Egypt, 10, 137 (1959).

17. H. O. Foulkes, J. London Math. Soc. 26, 132 (1951).

18. H. O. Foulkes, Trans. Roy. Soc. (London), 246, 555 (1954).

19. M. J. Newell, Quart. J. Math. (Oxford), 2, 161 (1951).

20. A.O. Usher, Can. J. Math., 28, 440 (1976).

21. O. Egecioglu and J. B. Remmel, Atomic Data and Nuclear Data Tables, 32, 157 (1985).

22. O.A. Nava, J. Comb. Theor. A46, 212 (1987).

23. S.C. Black and R. J. List, Eur. J. Combin., 10, 111 (1989).

24. C. Carre and J. Y. Thibon, Adv. Appl. Math., 13, 390 (1992).

25. T. Scharf and J. Y. Thibon, Cr. Acad. Sci I-Math, 13, 983 (1992).

26. J.P. Brennan, Commun. Algebra, 21, 1029 (1993).

27. M.A. Salam, Utilitas Mathematica, 50, 33 (1996).

28. M. Brion, J. London Math. Soc., 52, 449 (1995).

29. T. Scharf, J. Y. Thibon and B. G. Wybourne,
J. Phys A-Math. Gen., 30, 6936 (1997).

30. C. Malvenuto C. Reutenauer, Discrete Math., 193, 225 (1998).

31. W.F. Doran, J. Algebr. Comb., 8, 253 (1998).

32. M. Yang, Discrete Math., 180, 391 (1998).

33. G. de B. Robinson, Representation Theory of the Symmetric Group, Edinburgh Univ. Press, Edinburgh, 1961.

34. I. M. Gel'fand, R. A. Minlos and Z. Ya. Shapiro, Representations of the Rotation and Lorentz Groups and their Applications, Pergamon Press ss, Oxford, 1963.

35. J. P. Elliott, Proc. Roy. Soc. A245, 128 (1958).

36. J. M. Blatt and V. F. Weisskopf, Theoretical Nuclear Physics, John Wiley, New York, 1960.

37. N. Hamermesh, Group Theory, AddisonWesley, Reading, Mass., 1962.

38. V. Vanagas and L. Y. Sabalauskas, Lietuvos fizikos rinkinys(Lithuanian Journal of Physics, in Russian) 8, 77 (1968).

39. J. A. Castilho Alcarás, J. Tambergs, T. Krasta, J. Ruža and O. Katkevičius, Fizika(Iuguslavia) 22, 101 (1990); IJMPE 6, 341 (1997); Braz. J. Phys. 27, 425 (1997); proceedings of the Nininth Nordic Meeting on Nuclear Physics, August 4-8, 1998, Jyvaskyla, Finland; J. Phys. G: Nucl. Part. Phys. 25, 925 (1999). 